\title{
A Review of AI and Data Science Support for Cancer Management
}

\author{
Authors: \\ E. Parimbelli, S. Wilk, R. Cornet, P. Sniatala, K. Sniatala, S.L.C. Glaser, I. Fraterman, A.H \\ Boekhout, M. Ottaviano, M. Peleg
}

\section{Corresponding author:}

Enea Parimbelli, Ph.D.

Post-doctoral research fellow

Department of Electrical, Computer and Biomedical Engineering

University of Pavia

Via A. Ferrata 5 - 27100 Pavia

enea.parimbelli@gmail.com

\begin{abstract}
Introduction - Thanks to improvement of care, cancer has become a chronic condition. But due to the toxicity of treatment, the importance of supporting the quality of life (QoL) of cancer patients increases. Monitoring and managing QoL relies on data collected by the patient in his/her home environment, its integration, and its analysis, which supports personalization of cancer management recommendations. We review the state-of-the-art of computerized systems that employ AI and Data Science methods to monitor the health status and provide support to cancer patients managed at home.

Objective - Our main objective is to analyze the literature to identify open research challenges that a novel decision support system for cancer patients and clinicians will need to address, point to potential solutions, and provide a list of established best-practices to adopt.

Methods - We designed a review study, in compliance with the Preferred Reporting Items for Systematic Reviews and Meta-Analyses (PRISMA) guidelines, analyzing studies retrieved from PubMed related to monitoring cancer patients in their home environments via sensors and selfreporting: what data is collected, what are the techniques used to collect data, semantically integrate it, infer the patient's state from it and deliver coaching/behavior change interventions.

Results - Starting from an initial corpus of 819 unique articles, a total of 180 papers were considered in the full-text analysis and 109 were finally included in the review. Our findings are
\end{abstract}


organized and presented in four main sub-topics consisting of data collection, data integration, predictive modeling and patient coaching.

Conclusion - Development of modern decision support systems for cancer needs to utilize best practices like the use of validated electronic questionnaires for quality-of-life assessment, adoption of appropriate information modeling standards supplemented by

terminologies/ontologies, adherence to FAIR data principles, external validation, stratification of patients in subgroups for better predictive modeling, and adoption of formal behavior change theories. Open research challenges include supporting emotional and social dimensions of wellbeing, including PROs in predictive modeling, and providing better customization of behavioral interventions for the specific population of cancer patients.

Keywords: Cancer, Decision Support System, Data Science, Data Integration, Patient Reported Outcomes, Quality of Life, Artificial Intelligence, Predictive modeling, Patient coaching 


\section{Introduction and background}

Computerized systems that support clinical decision making and action management often use artificial intelligence (AI) methods to represent, mine, and reason with medical knowledge. These systems demonstrate human-like artificial intelligence for supporting medical diagnosis, prevention, and care [1]. The knowledge that forms the basis for the AI-based support is acquired from clinical guidelines, evidence-based studies or experts, or it can be mined from electronic health records (EHRs) [2].With the explosion in the availability of health data, collected in EHRs or acquired via wearable sensors, the scope of AI methods in medicine [3] has widened to include such data-centric methods that may be used to generate medical knowledge that drives the AI-based systems.

Data is at the heart of data science (DS) methods, which address all stages of the data life cycle [4]. These stages encompass data generation and collection; data processing, integration, storage, and management; data analysis; and finally, data visualization and interpretation; these stages are often grouped into a smaller number of higher-level categories [5]. We grouped them into four categories: data collection (covering data generation and collection); data integration (covering processing, integration, storage, and management); data analysis, and in particular predictive modeling; and results communication (covering visualization and interpretation) - See Figure 1.

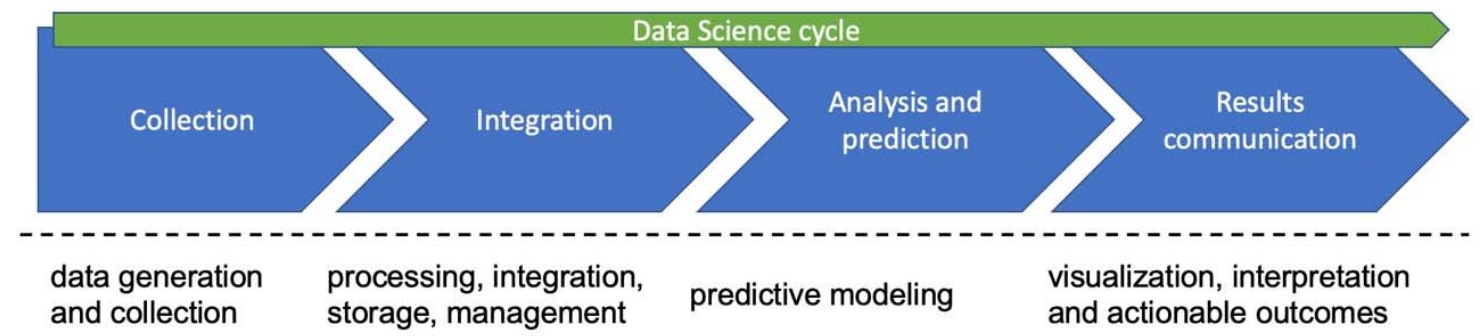

Figure 1 - Data Science cycle and categories defining the scope of the literature review. Under the dashed line, mapping of our grouped categories to the taxonomy of phases proposed in [4].

The use of DS methods is already demonstrating their potential for transforming medical care, including radiology[6], surgery [7] and laboratory medicine [8]. The focus of this review is on the use of DS for developing AI-based systems supporting patients managed at home and the healthcare providers caring for them. Such a topic has been addressed before in a workshop held in 2017 on innovations in emerging technology, user-centered design, and data analytics for behavioral health [9]. The workshop focused on wearable and mobile technologies being used in clinical and research contexts, with an emphasis on applications in mental health and health behavior change. The workshop included panels on mobile sensing, user experience design, statistics and machine learning, and privacy and security, and led to the identification of research directions for this important and emerging field of applying digital approaches to behavioral health.

Our review focuses in particular on use of DS methods to support care of cancer patients managed at home. After the primary intervention, most cancer patient survivors are managed at home, facing long-term (sequelae) treatments, making the disease comparable to a chronic 
condition [10]. Despite their benefit, strong therapeutic regimens often cause toxicity, severely impairing quality of life. This may decrease adherence to treatment, thus compromising therapeutic efficacy. Also due to age-related multimorbidity, patients and their caregivers develop emotional, educational and social needs. The CAncer PAtients Better Life Experience Horizon 2020 project (CAPABLE, H2020 875052) will develop a cancer patient coaching system with the objective of facing these needs, in the effort to fully exploit the potential of Data Science to support cancer care and bring it to patients' homes.

Following the data life cycle approach described above, in this article, we review the literature related to DS methods that cover the entire data life cycle, including: (a) data collection (i.e. monitoring cancer patients in their home environments via sensors and self-reporting);(b) semantic data integration; and based on it provide (c) inference and prediction of the patient's state; and (d) deliver visualization, interpretation, and communication, which for cancer patients monitored at home, is in the form of coaching, including behavior change interventions.

CAPABLE will indeed use a DS approach in order to provide support to patients and clinicians in two different pathways (Figure1); after (1) collecting data and (2) semantically integrating it, these data can be used to (3) build predictive models that help clinicians infer the patient state and (4) drive patient coaching systems that provide patients with personalized evidence-based recommendations on how to improve their wellbeing. Because the data science cycle encompasses all of these four areas, they define the scope of the review [11]. The findings of the analyses in this review will be used to identify best practices and open research challenges that go beyond state of the art.

\section{Methods}

\subsection{Search strategy}

We designed a review study in compliance with the Preferred Reporting Items for Systematic Reviews and Meta-Analyses (PRISMA) guidelines [12]. We conducted a literature search of the PubMed repository for studies published in the last 5 years (2014-2019) describing AI-enabled systems focusing on the monitoring and decision support of cancer patients. The last search was conducted on March 15, 2020. Our search strategy consisted in the union of four search queries, one addressing each of the identified sub-topics: data collection, data integration, AI/prediction models and coaching systems. The queries were built looking for search terms in TextWord [tw] (which includes title, abstract, Mesh terms and author-identified keywords) in order to increase query recall compared to searching just the title [ti] [13]. Queries included (system [tw] OR app [tw] OR application [tw]) in order to identify research that produced some sort of computerized clinical decision support system. Finally, specific terms for each of the four subtopics were added to scope down each of the specific search queries. We report below the query used to collect papers dealing with coaching systems for cancer patients, while the complete search strings employed in the article identification phase are reported in supplementary Table s1. After collecting the results in a Zotero library (www.zotero.org), duplicates were removed. Titles and abstracts of each paper were screened using Abstrackr [14], and irrelevant articles removed before full-text analysis and quality appraisal. 
cancer[tw]

AND (system[tw] OR app[tw] OR application[tw])

AND ((coach[TW] OR coaching[tw]) OR ("behavior change"[tw] OR "behavioral change" [tw]))

\subsection{Eligibility criteria}

We considered articles written in English and published in peer-reviewed journals or conference proceedings in the last five years. Upon initial screening of title and abstract we excluded articles meeting any of the following criteria:

1) Papers dealing exclusively with imaging, genetics or other -omics

2) Papers on statistical survival analysis

3) Papers not dealing with home/outpatients

4) Papers not including the development of a computer-based DSS or computerized behavioral interventions

5) Papers describing protocols for future studies

6) Papers discussing patients being managed for undiagnosed cancer (i.e., no screening tools, even if they are computer-based)

7) Papers on novel drug development, prediction of response to a certain drug, drug-drug interaction, and drug repurposing

8) Papers dealing with animal models (i.e. no human patients)

Note that review articles were excluded from the review itself, but were used to inform the discussion in the present article. Similarly, the references of such review papers were screened to identify relevant articles which, when found, where added to the list of articles considered in the review. The complete list of articles analyzed in this review appears in the Bibliography.

\subsection{Quality appraisal}

All manuscripts included in the present review underwent a formal quality appraisal following the critical appraisal tool from CASP [15]. We selected the CASP qualitative checklist, as it resulted the most widely applicable to the set of articles we reviewed. The results of the quality appraisal have been included in the supplementary material as supplementary Table s2.

\section{Results}

Figure 2 reports the PRISMA flow-diagram of the systematic review. A total of 179 papers were included in the full-text analysis. 70 articles were excluded after accessing their full text, for the reasons listed in Table 1. 
Records identified through database

searching

$(n=318+136+292+85=831)$
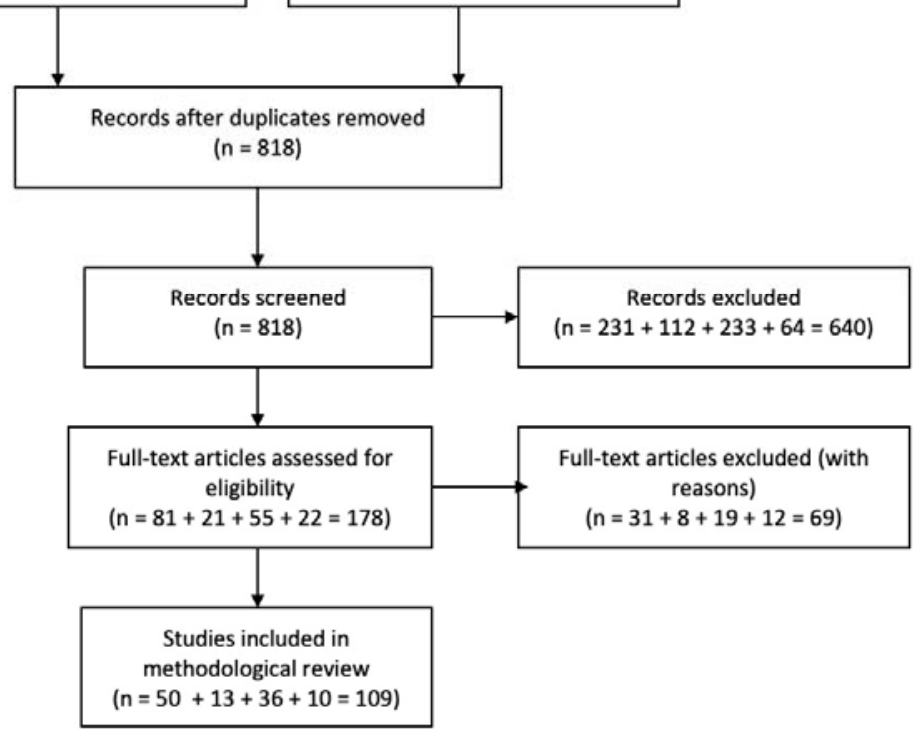

Figure 2 - PRISMA flow diagram. The numbers reported at each stage before the total refer to the data collection, data integration, predictive modeling and coaching subtopics.

Table 1. Number (n) of articles that were excluded, after full-text analysis, for each subtopic of the review and the reasons for exclusion.

\begin{tabular}{|c|c|c|c|c|}
\hline Reason for exclusion & Data Collection & Data Integration & Predictive modeling & Coaching \\
\hline $\begin{array}{l}\text { Focus was on a different } \\
\text { topic }\end{array}$ & $\begin{array}{l}\text { (10) } \\
1 \text { focused on the } \\
\text { design of a general } \\
\text { architecture for } \\
\text { speeding up the } \\
\text { acquisition of PROs } \\
3 \text { focused on the needs } \\
\text { assessment before } \\
\text { developing and } \\
\text { designing the eHealth } \\
\text { tool. } \\
1 \text { is about a result of a } \\
\text { cancer treatment } \\
\text { (lymphedema) but not } \\
\text { on cancer monitoring } \\
\text { itself }\end{array}$ & $\begin{array}{l}\text { (6) } \\
1 \text { focused on security } \\
5 \text { focused on patient- } \\
\text { reported outcomes }\end{array}$ & $\begin{array}{l}\text { (14) } \\
8 \text { dealt with } \\
\text { screening/diagnosis } \\
\text { of cancer } \\
4 \text { statistical } \\
\text { hypothesis testing } \\
1 \text { focused only on } \\
\text { genetic data } \\
1 \text { about chemistry } \\
\text { and drug discovery }\end{array}$ & $\begin{array}{l}\text { (5) } 1 \text { dealt } \\
\text { with } \\
\text { organizati } \\
\text { onal } \\
\text { integration } \\
\text { with } \\
\text { standard } \\
\text { care, } 1 \\
\text { focused on } \\
\text { the } \\
\text { language } \\
\text { used in } \\
\text { available } \\
\text { apps, 1 } \\
\text { described } \\
\text { coaching }\end{array}$ \\
\hline
\end{tabular}




\begin{tabular}{|c|c|c|c|c|}
\hline & $\begin{array}{l}1 \text { focuses on the } \\
\text { graphical } \\
\text { representation of } \\
\text { medical data } \\
1 \text { focuses on patient } \\
\text { feedback about 'user- } \\
\text { friendliness' of the } \\
\text { system } \\
1 \text { focuses on software } \\
\text { optimisation } \\
1 \text { focused on physical } \\
\text { exercise } \\
1 \text { deals with paediatric } \\
\text { patients }\end{array}$ & & & $\begin{array}{l}\text { by humans } \\
\text { over the } \\
\text { phone, } 1 \\
\text { discussed } \\
\text { the design } \\
\text { of a } \\
\text { hypothetic } \\
\text { al app, } 1 \\
\text { described } \\
\text { design } \\
\text { process of } \\
\text { an app } \\
\text { presented } \\
\text { in another } \\
\text { (included) } \\
\text { paper }\end{array}$ \\
\hline $\begin{array}{l}\text { Paper did not detail the } \\
\text { methods or was an } \\
\text { abstract }\end{array}$ & (4) & (1) & (3) & (2) \\
\hline Paper was a review & (2) & (1) & (2) & (2) \\
\hline $\begin{array}{l}\text { Paper described a } \\
\text { protocol for a future } \\
\text { study }\end{array}$ & (15) & & & (3) \\
\hline Total \#excluded articles & 31 & 8 & 19 & 12 \\
\hline
\end{tabular}

\subsection{Data collection}

In order to monitor health status following cancer treatment, different types of data should be collected; in addition to physician-provided clinical data, laboratory tests, and imaging results, the data should include patient-provided data and sensor data. Patients may complete patientreported outcomes (PROs) to report data actively, without an interpretation of patients' response by a clinician or anyone else. PROs are usually collected through (online) questionnaires. They assess different constructs, consisting of disease or treatment related symptoms, (general) health status or health-related quality of life (HRQoL) outcomes. Another form of collecting data from patients is via sensors. Mobile sensors may be used to collect vital signs from patients in their home environment.

\subsubsection{Methods of collecting data from patients}

The majority of papers in the Data Collection category (49 of 50) collected data via manual input through electronic PROs implemented inside mobile apps running on smartphones, tablets or using dedicated websites. More popular collection methods were mobile apps, used in 59\% of the papers, vs. websites, used in $41 \%$ of the papers. In ten papers, sensor data was automatically gathered using sensors embedded in smart bands, smartwatches and other electronic devices. Smartphones were used mainly to gather data from dedicated sensors, which measured physical 
activities (step count) and vital signs (weight, blood pressure, oxygen saturation, pulse, heart rate and temperature). Among the reviewed papers, "built-in" smartphones' sensors were not used to collect data.

\subsubsection{Patient Reported Outcomes}

Linton et al. [16] reviewed 99 self-report measures for assessing well-being in adults and categorized them into six themes; the four dimensions from patients' life mentioned above, and in addition, spiritual well-being and personal circumstances. According to Linton et al.:

Physical well-being "refers to the quality and performance of bodily functioning, including experiences of pain and comfort.

Social well-being "concerns how well an individual is connected to others in their local and wider social community".

Mental well-being "assesses the psychological, cognitive and emotional quality of a person's life", including the thoughts and feelings that individuals have about the state of their life, and a person's experience of happiness".

Activities and functioning "is the behaviour and activities that characterise daily life and our ability to undertake these tasks".

Spiritual well-being "is concerned with meaning, a connection to something greater than oneself and in some cases faith in a higher power".

Personal circumstances "are related to the conditions and external pressures that an individual faces, including financial security".

Patient Reported Outcomes (PROs) are usually collected online, through questionnaires, and assess different constructs, consisting of disease or treatment related symptoms, (general) health status or health-related quality of life (HRQoL) outcomes. HRQoL is fundamentally subjective and multidimensional. HRQoL is measured from the perspective of the patient and covers a range of dimensions from patients' life, including physical-, social-, emotional-/mental-, and functioning [17]. The European Platform of Cancer Research (EORTC) adds that HRQoL covers the subjective perceptions of the positive and negative aspects of cancer patients' disease symptoms and side effects of treatment, and stresses the cognitive aspect of functioning [18].

PROs focusing on HRQoL were measured generally before and after the intervention instead of during the use of the eHealth tool, whereas PROs regarding symptom monitoring were collected during the intervention and treatment. Computer Adaptive Testing (CAT) can be applied to shorten the number of questions being asked. CAT is a method to select item sets for individual patients based on a patient's responses to previous items. The algorithm then selects new items from item banks to maximize the obtained information. Therefore, fewer items are needed to precisely measure patients' scores. Patient scores can also be directly comparable, even when not answering the same item lists, which is done by item response theory (IRT) methods [19].

In most included papers, combinations of questionnaires were used to collect patient-reported data. Most articles used at least one validated PROs questionnaire and comprised large sample sizes (up to 4345 patients). Patient populations were biased since recruited patients were a-priori in favor of using an eHealth application. PROs questionnaires used for data collection in this review are listed in Table 2. This table presents the reviewed PROs according to well-being 
medRxiv preprint doi: https://doi.org/10.1101/2020.08.07.20170191; this version posted February 11, 2021. The copyright holder for this preprint (which was not certified by peer review) is the author/funder, who has granted medRxiv a license to display the preprint in perpetuity.

It is made available under a CC-BY-NC-ND 4.0 International license .

dimensions that were defined by Linton et al. [16]. Furthermore, this table presents additional PROs on symptoms. PROs were added to this table when used in 3 or more papers.

Table 2. Patient Reported Outcomes (PROs) questionnaires used in the studies. Questionnaire instruments listed in the table are all validated and have been used by at least 3 studies.

\begin{tabular}{|c|c|c|c|c|c|c|c|c|}
\hline \multirow{2}{*}{$\begin{array}{l}\text { Questionn } \\
\text { aire }\end{array}$} & \multicolumn{7}{|c|}{ Type of data collected } & \multirow{2}{*}{$\begin{array}{l}\text { \#Articles } \\
(\% \\
\text { papers } \\
\text { used })\end{array}$} \\
\hline & $\begin{array}{l}\text { Global } \\
\text { well- } \\
\text { being }\end{array}$ & $\begin{array}{l}\text { Mental } \\
\text { well- } \\
\text { being }\end{array}$ & $\begin{array}{l}\text { Social } \\
\text { well- } \\
\text { being }\end{array}$ & $\begin{array}{l}\text { Physical } \\
\text { well- } \\
\text { being }\end{array}$ & $\begin{array}{l}\text { Activities } \\
\text { and } \\
\text { functionin } \\
\mathrm{g}\end{array}$ & $\begin{array}{l}\text { Patient- } \\
\text { reported } \\
\text { symptom } \\
\text { S }\end{array}$ & $\begin{array}{l}\text { \#items in } \\
\text { question } \\
\text { naire }\end{array}$ & \\
\hline $\begin{array}{l}\text { EORTC } \\
\text { QLQ-C30 }\end{array}$ & $\mathrm{x}$ & $\mathrm{x}$ & $\mathrm{x}$ & $\mathrm{X}$ & $\mathrm{X}$ & $\mathrm{x}$ & 30 & $\begin{array}{l}14 \\
{[20-33]} \\
(28 \%)\end{array}$ \\
\hline PROMIS & & $\mathrm{x}$ & $\mathrm{x}$ & $\mathrm{x}$ & & & $\begin{array}{l}4-20 \\
\text { (short } \\
\text { forms), } \\
\text { but holds } \\
\text { multiple } \\
\text { item } \\
\text { banks }\end{array}$ & $\begin{array}{l}7 \\
{[22,26,3} \\
4-38] \\
(16 \%)\end{array}$ \\
\hline $\begin{array}{l}\text { EQ-5D- } \\
5 \mathrm{~L}\end{array}$ & & $\mathrm{x}$ & & $\mathrm{x}$ & $\mathrm{X}$ & & 5 & $\begin{array}{l}4 \\
{[22,28-} \\
31] \\
(8 \%)\end{array}$ \\
\hline $\begin{array}{l}\text { SF- } \\
8 / 14 / 36\end{array}$ & & $\mathrm{x}$ & $\mathrm{x}$ & $\mathrm{x}$ & $\mathrm{x}$ & & $8 / 14 / 36$ & $\begin{array}{l}4 \\
{[33,39-} \\
41] \\
(8 \%)\end{array}$ \\
\hline HADS & & $\mathrm{x}$ & & & & & 14 & $\begin{array}{l}3[42- \\
45] \\
(6 \%)\end{array}$ \\
\hline FACT-G & & $\mathrm{x}$ & $\mathrm{x}$ & $\mathrm{x}$ & $\mathrm{x}$ & & 27 & $\begin{array}{l}3 \\
{[43,46,4}\end{array}$ \\
\hline
\end{tabular}




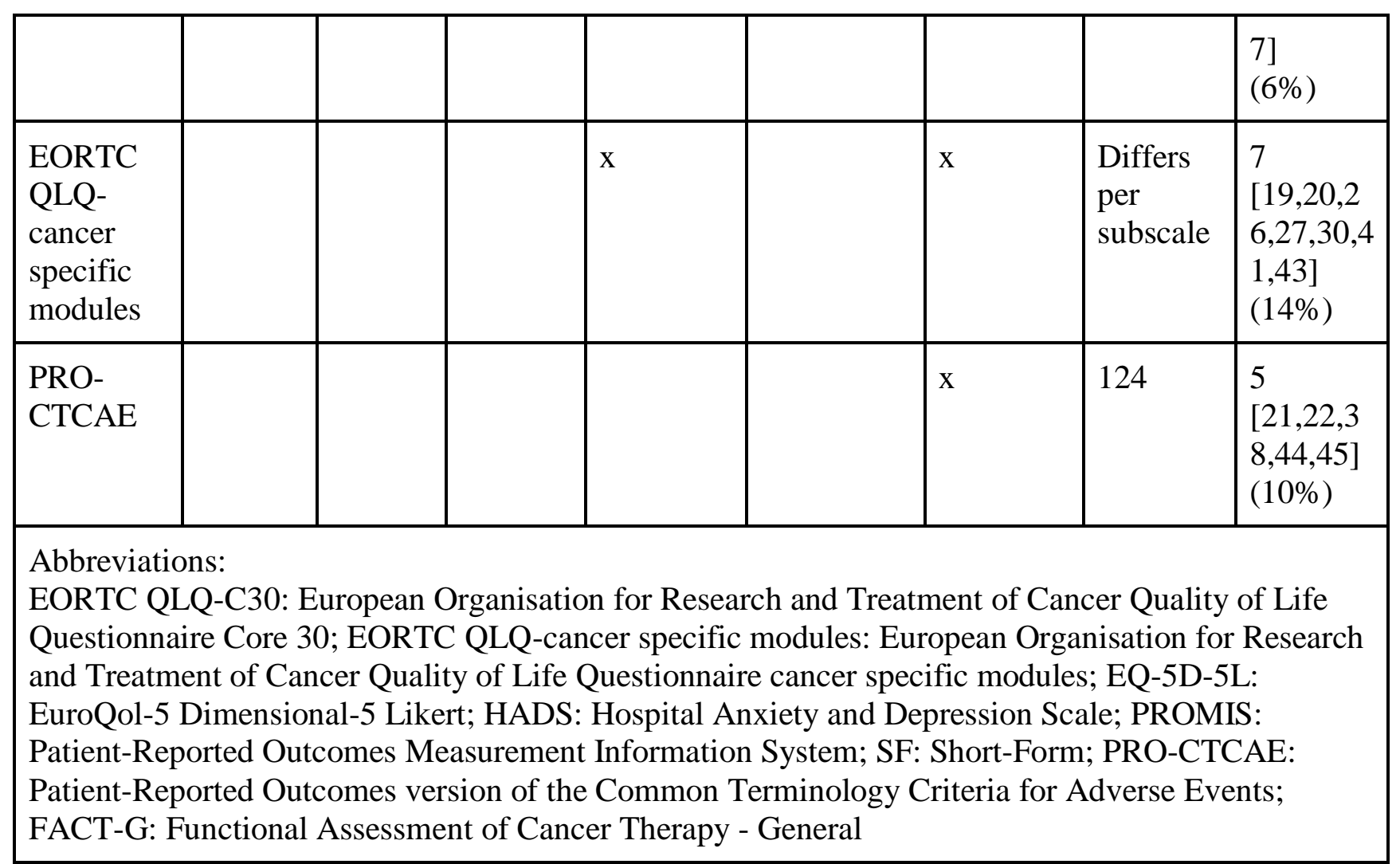

\subsubsection{Patient Reported Outcomes: health-related quality of life}

In this review, the most commonly used validated PRO questionnaire for collecting HRQoL data is the EORTC QLQ-C30, used in 28\% of the 50 included papers [20-33]. It is a European widely used core generic questionnaire for cancer patients, and comprises most dimensions of wellbeing as described by Linton et al. [16]1, excluding spiritual wellbeing and personal circumstances. In fact, these dimensions were not captured by any of the PROs used in the reviewed papers. The Functional Assessment of Cancer Therapy - General (FACT-G), also a cancer-specific HRQoL questionnaire is more frequently used in America, but was less frequently used for data collection -- in $6 \%$ of included papers [43,46,47]. The second most used questionnaire in this review was Patient-Reported Outcomes Measurement Information System (PROMIS), a set of person-centered measures (enabled by CAT and IRT), used in $16 \%$ of the papers [22,26,34-38]. Third most used (8\%) questionnaire was the EQ-5D-5L [22,28-31] questionnaire that can be used to compute Quality-adjusted Life Years (QALY's), a generic measure of disease burden, including both the quality and the quantity of life lived. Therefore, the EQ-5D-5L questionnaire can be used for cost-effectiveness analyses.

\subsubsection{Patient Reported Outcomes: symptoms}

Several PRO questionnaires were used to collect data regarding patient reported symptoms. The EORTC QLQ-cancer specific modules were used to collect cancer-specific patient-reported symptoms in 7 out of 50 included papers $[19,20,26,27,30,41,43]$. These modules are specific to 
tumour site, treatment modality, or a HRQoL dimension, to be administered in addition to the core questionnaire (EORTC QLQ-C30) [44]. Furthermore, the Patient-Reported Outcomes version of the Common Terminology Criteria for Adverse Events (PRO-CTCAE) was a commonly used $(10 \%)$ questionnaire to capture information on symptomatic adverse events $[21,22,38,44,45]$. The PRO-CTCAE Item Library includes 124 items representing 78 symptomatic toxicities drawn from the Common Terminology Criteria for Adverse Events (CTCAE), reported by patients [48].

\subsubsection{Collection of patient data in their home environment using sensors}

In this section we discuss the use of sensors to automatically collect additional data (typically vital signs) in the patient home environment. Such data complements PROs captured with questionnaires described in the previous section. Since using sensors imposes no (or limited) burden on the patient (no manual data entry), such modality can be used for frequent and longterm data collection. Reviewed papers described several systems of diversified complexity in terms of the range of employed sensors and the scope of collected data. Group 1 systems consist of a mobile device (smartphone or tablet) connected to a smartband or bracelet (an app usually performs preprocessing and transmits data to a backend; interestingly, none of the described systems relied on sensors embedded in a smartphone). Group 2 is further extended by external sensors (like activity tracker or accelerometers attached to the body), while the smartphone still acts as a hub where the data is collected, processed and transmitted. Group 3 includes systems with standalone devices, which work independently (sometimes) without smartphones. They are more complex and utilize specialized devices like EEG/EMG monitors.

The first group includes simple systems where a smartphone or a tablet is expanded with a wearable device, typically a consumer smartband. Soh et al. [22] described an app providing self-monitoring for gastrointestinal cancer patients. In addition to electronic questionnaires, patients were equipped with wearable bands connected to the app via Bluetooth and used to collect physical activity (PA) information, such as steps count and burned calories. A similar solution was presented by Sun et al [49]. It employed online questionnaires to record HRQoL and smartbands (Vivofit 2 and Garmin Ltd) to monitor PA. PA was monitored by checking the number of patient's daily steps during recovery. Data from the smartband was being collected 3 to 7 days before surgery, during the patients' stay at the hospital and approximately 2 weeks after their release from the hospital. Finally, Pavic et al. [32] described a system where patients were provided with a smartphone with preinstalled "Activity Monitoring', app and a commercial sensor-equipped bracelet (Everion). The app recorded motion sensor data (GPS, acceleration), phone call statistics (anonymized), and selected vital signs measured by the bracelet, which was automatically connected to the smartphone via Bluetooth Low Energy. The collected data included step count, step speed and resting heart rate (RHR). Encrypted data was sent once daily to a secured server.

The second group includes systems with additional sensors, which transfer collected data to the smartphones. Timmerman et al. [50] described a system for collecting self-reported symptom severity, mood, medication use and weight. Along with the smartphone, the patient was also equipped with three sensors attached to the body -- an accelerometer (to measure PA). heart rate sensor and oxygen saturation sensor (PPG). Data collected by the wearable devices and the 
symptom scores were summarized into graphs and made available to doctors and patients. Due to adding wearable sensors, results collected via the symptom questionnaires could be confronted with the collected vital signs. Nápoles et al. [38] presented a solution where a commercial-grade activity tracker was used together with the app to monitor and display progress toward a personalized daily steps goal.

The third group of systems includes solutions where multiple sensors are used in order to collect the required information. Rahman et al. [26] presented a Secure Occupational Therapy (OT) Framework, which aims at HRQoL monitoring. The proposed system uses multiple sensors to detect activities of daily life. The OT movements are mapped to QoL measurements metrics. The collected data can be shared with an oncologist or palliative care unit for real-time decision support. The hardware includes a set of IoT devices such as an LIFX light bulb, door lock, Emotiv EEG device, MYO EMG device, LEAP motion hand gesture tracking sensor, Kinect body gesture tracking sensor, and Eye Tribe pupil tracing sensor. The captured data is transmitted through the secure digital wallet to distributed apps (DApp). These apps apply the privacy and encryption model and offload the data to the edge network for further storage and sharing. Authors proposed DApps for patients, family members, cancer therapists, a secure online doctor on demand, hospital personnel, and medical IoT devices to be able to record the data of cancer patients, caregivers to be able to be in touch with the patients, and data management planning.

We note that using Kinect (and similar) sensors to monitor and provide feedback to patients performing intervention or rehabilitation exercises is an upcoming trend in healthcare. However, the joint positions measured by the Kinect sensor are often unreliable, especially for joints that are occluded by other parts of the body. In addition, users' motion sequences differ significantly even when doing the same exercise and are not temporally aligned, making the evaluation of the correctness of their movement challenging. Chiang et al. [51] presented a Kinect-based intervention system, which can guide the users to perform the exercises more effectively.

Metcalf et al. [52] presented a health care application to provide more extensive patient education and more thorough perioperative monitoring. Patients with home Wi-Fi access were equipped with tablets preloaded with the health care application (m.Care), an accelerometer and additional equipment for measuring selected vital signs. Specifically, patients participating in the study were provided with the wireless weight scale, the upper arm blood pressure monitor, and the external wireless pulse oximeter. All of these devices were Bluetooth capable and could sync directly to the m.Care application. Participants were asked to perform 5 vitals assessments daily (weight, blood pressure, oxygen saturation, pulse and temperature) for 5 days preoperatively and daily upon hospital discharge until the first postoperative visit.

As can be seen from the above examples, the majority of systems measure the PA of patients. This information is collected based on step count, step speed, which are collected by accelerometers $[22,32,50]$ and burned calories [22]. Solutions that are more complex measure heart rate, oxygen saturation [50] or weight, blood pressure, oxygen saturation, pulse and temperature [52]. There are also Kinect-based solutions, which can help patients to perform the recommended physical exercises $[22,51]$. 


\subsection{Data integration}

Data integration is needed when data are partitioned horizontally (i.e., when the same attributes are stored in multiple datasets for different individuals) and/or vertically (i.e., when attributes about individuals are spread out over multiple databases). Several of the reviewed papers highlight the need to integrate data from different organizations and systems to allow creating a big-data cohort, in terms of volume and variety of data. Such cohorts could be analyzed statistically [53], can allow clinical study data to be searched and compared, or can be used to create more accurate predictive models using machine learning methods[53-55]. Other works focus on the need to apply generic models of clinical decision support onto patient data in order to generate patient-specific recommendations [56,57].

Our review focuses on methods for the data integration task. The methods used in the papers that we reviewed leverage patient data standards (information models) or ontologies/terminologies to facilitate the data integration task. Third, integration facilitated by machine-learning methods is addressed.

\subsubsection{Data integration via information models}

The review paper by Bodenreider [58] stresses that HL7 standards support information exchange by providing an information model for different types of patient data, such as observations, medication requests, procedures, and encounters. Such HL7 classes include attributes for specifying the time stamp, the focus of messages (e.g., (e.g., blood pressure observation, Gemcitabine medication request) according to controlled terminologies such as ICD, LOINC, SNOMED CT and RxNorm, and more specialized attributes, depending on the FHIR class.

Indeed, several of the data integration systems that we have reviewed rely either on simple HL7 messages [59] or on the HL7 Fast Healthcare Interoperability Resources (FHIR) standard $[53,56,60]$ to provide semantics to the data that is integrated and allow standardized access to it. In some works, the databases that are accessed via FHIR-APIs, already provide clinical semantics that are richer than simple storage of medical terminology codes. For example, in KETOS [53] an extract, transform, load (ETL) process exports data from EHRs or data warehouses into an Observational Medical Outcomes Partnership (OMOP) common data model (CDA) database. In the work of Ulrich et al. [61], consortium data is uploaded into an i2b2 ('Informatics for Integrating Biology and the Bedside') database. Furthermore, that framework supports the definition of relations between individual data elements and their specification with the help of a suggestion system that uses the LexEVS terminology system of the National Cancer Institute. In [62], the authors develop and leverage a repository of medical data models, represented using CDISC ODM (Clinical Data Interchange Standards Consortium - Operational Data Model https://www.cdisc.org/standards/data-exchange/odm) format.This repository enables manual semantic annotation of data models, as well as construction of data sets that comply to existing data models.

Still, data integration using proprietary information models occurs. For example, Hill-Kayser et al. [57] developed an ETL process to populate LIVESTRONG Survivorship Care Plans with Epic EMR data so that these care plans become patient-specific. 
Some systems also use other standards. For example, [56] describes instantiation of a Bayesian Network for a patient using a combination of Arden Syntax Medical Logic Modules that specify the logic of the required processing of data. The "curly brace" statements in Arden Syntax describe the needed data elements by calling a FHIR resource that refers to the patient ID or a SNOMED code for clinical findings. Ulrich et al. [61] use the ISO 11179-3 metadata repository standard. Metadata about data elements is represented according to the FHIR standard. For example, Case Report Forms are represented as a FHIR questionnaire. The FHIR-based processing allows exchange of data elements with clinical and research IT systems as well as with other metadata systems.

\subsubsection{Integration of genomics and EHR data}

While the integration challenge often involves reaching consensus on similar data elements represented differently, either in different information models, or using different vocabularies, an additional challenge is the integration of vertically partitioned data of different modalities, coming from different realms, such as genomic data and clinical data. Several of the reviewed papers discuss this emerging need for integrating genomic (omics) data and clinical and epidemiological (non-omics) data. Integration of omics and non-omics $(\mathrm{OnO})$ data shows promise in improving predictive models, phenotyping patients and finding clinical risk factors. Integration of cancer genomic data with EHR systems could also improve clinical decision making and the use of personalized care. However, efforts are still scarce as integration of OnO data poses a range of challenges, such as the heterogeneous nature of clinical data and the lack of consistent standards for both genomic and clinical data. López de Maturana et al. [63] reviewed attempts of OnO data integration in clinical and epidemiological studies. Although few of their reviewed studies explicitly address this type of modeling, joint modeling approaches are recommended by López de Maturana et al. [63] for integration of large-scale OnO data. Joint modeling approaches capture a larger complexity than the modeling approaches more frequently seen such as conditional or independent modeling. Additionally, joint modeling accounts for the correlation structure between the two data types.

Mihaylov et al. [64] present a framework for this joint modeling of clinical and omics data. A network is presented with the aim to predict survival time. Utilizing semi-structures for each data type (such as clinical, expression and mutation data), relations are found that represent the internal network. Enrichment of these data and relations is possible by linking data to external domain knowledge sources. Mihaylov et al. show the utility of semantic OnO data integration as survival time prediction models bear improved results using the relational OnO data network compared to separate omics and non-omics survival prediction.

Focusing on integrating genomic data with EHR systems, Warner et al. [65] discuss the status (as of 2016) and the challenges. Although well-established nomenclatures exist for omics data, a lack of consistent modeling standards prevents integration of omics laboratory results into EHRs. Currently, laboratory results are included in PDF format which does not allow for any secondary use of data or for clinical decision support. Deemed as most useful of the emergent solutions is the use of application programming interfaces (APIs) or RESTful web services. Particularly, SMART on FHIR clinico-genomics apps were highlighted as solutions that incorporate the presentation and contextualization of genetic test results into the clinician workflow. 


\subsubsection{Data integration via ontologies and vocabularies}

The review paper by Bodenreider [58] provides an overview of how ontologies can be used for data integration. First, in the warehousing approaches, controlled vocabularies can be used to describe the focus of data items in local sites, facilitating their later integration. The integration can span data sets with different content that may be combined. For example, LOINC has been used for integrating laboratory data with adverse events, the Foundational Model of Anatomy for the integration of genomic information sources, and SNOMED CT for the integration of disease and pathway information.

Second, mediation-based approaches use ontologies for defining a common global schema for data elements (common data elements, CDE) and mappings between the global schema and local schemas are then defined. This allows composing queries to the local schemas in terms of the common schema.

The data integration approaches from recent years use a combination of both approaches. Several papers $[53,54,61,66-68]$ present ETL database functions that allow pulling data from a source database and placing it into a target database. The first three of these papers define manual processes by which local sites define mappings between source data elements (SDE) to a set of needed common data elements (CDE). Use of controlled vocabularies such as SNOMED CT and LOINC facilitates the agreement. Similar to the terminology-suggestion system of Ulrich et al [61] described in Section 3.2.1, Mate et al [68] support term matching further via an algorithm that supports fuzzy matching of terms, utilization of synonyms, and sentiment tagging to suggest mapping of SDEs to CDEs. A final step of data alignment replaces the source value sets with those from the target terminology, as well as converts between different data types according to expert-curated mapping rules.

\subsubsection{Data integration supported by machine learning}

While the methods described above have a focus on manual or rule-based integration based on ETL-procedures, increasingly machine learning methods are applied for data integration.

Mariette et al. [55] propose an approach that uses combined kernels in kernel self-organizing maps (KSOM) to cluster and visualize multi-omics breast cancer datasets. This clustering is relevant to discriminate between different breast cancer subtypes and to identify their relations and has the strength that it can be performed in an unsupervised manner, removing much of the burden of integration based on information models and ontologies.

Isoviita et al. [54] present an open-source, cloud-based machine learning system where datasets from multiple (live) sources (EHR databases and research databases) are integrated using extract, transform, load (ETL) processes and melded into a single database, but with minimal transformations. These merged but heterogeneous data are used for the training of ML predictive models. In this case study where primary therapy outcomes in high-grade serous ovarian cancer were predicted, results again demonstrated the benefit of combining information from multiple sources.

These papers show that machine learning enables analyses on integrated data without the need to perform supervised transformation of data, which is generally a resource-intensive task. 


\subsection{Predictive modeling}

\subsubsection{Input data for predictive modeling}

One of the main preconditions for building an accurate and reliable prediction model is the availability of high-quality, large-enough dataset to serve as the model training set. This is true in cancer, as well as in other medical domains. In addition to the choice of a particular ML algorithm, the performance of a predictive model largely depends on what input data has been used for training. The availability, promoted in recent years, of publicly accessible databases and registries focusing on cancer patients has proven to be a major driver of interest for studies on predictive modeling of cancer outcomes. One outstanding example is Surveillance, Epidemiology, and End Results (SEER) database, which has been used in a number of the analyzed articles [69-75]. On the other hand, availability of one own organization original data (e.g. from EHR) can constitute a significant competitive advantage with the possibility of accessing a wider array of patient data variables, and combining them for improved knowledge discovery and ultimately better predictive performance for specific populations. For example Soguero-Ruiz and colleagues [76] were able to leverage EHR clinical notes to build a model to predict anastomosis leakage in patients with colorectal cancer starting from NLP-extracted features. In another study [77] the same authors complemented EHR free text data with detailed labs and vital signs data to improve adverse event prediction. Again, Alabi et al. [78,79] used data coming from a federation of hospitals (in Finland and Brazil respectively) to build prognostic models for oral cancer tumor recurrences.

Large, publicly available datasets can provide the optimal conditions when size of the training data, standardization of variables and reproducibility (also comparability to other published research) of results are considered of the utmost importance. However, dealing with an original, unpublished dataset, usually owned by partnering clinical organizations, can provide a competitive edge in originality of the research and access to an extended number of independent predictors (e.g. detailed labs, or -omics data) that might prove useful for achieving the best predictive performance.

An additional finding that emerged from the analysis of the articles is that current research in predictive modeling should strive to generate generalizable models (i.e. models that perform well when applied to cohorts different from the one used for the original development and validation). Thus the use of a restricted set of easily collectable predictors is to be regarded as a desirable feature. One example can be found in Oliveira et al. [75] where a set of only 6 features from the SEER dataset proved effective in predicting survival of colorectal cancer patients at different time points (year 1,2,3,4 and 5). A notable characteristic of such model is the use of a dynamic set of predictors that evolve over time, to achieve best performance. The fact that the knowledge elicited by ML models should be validated over time is also a focal point to promote model generalizability, especially after some years from the original model elicitation. Indeed Kleinen and colleagues [72] advocate for knowledge embedded in predictive models for breast cancer to be updated every ten years to maintain good performance. 


\subsubsection{The quest for more complex machine learning models: ensembles, deep learning and the} like

Once the issue of possessing a good input dataset to train a well-performing model is solved, the next question researchers in this field face is what ML algorithm to use. In recent years, in light of the big-data era, there has been some debate [80-82] on whether cutting-edge ML models could deliver better performance than well-established statistical methods (e.g. Cox regression for survival analysis) that have been traditionally employed in cancer outcomes research. Whereas most of the articles analyzed employ a quite traditional approach where an array of ML algorithms is applied looking for the best-performing one, some notable exceptions occur. Deep Learning (DL) is for sure one of the paradigms that is drawing attention especially in cases where large and unstructured (e.g. -omics) data is available. The application of DL to medical predictive modeling is out of the scope of the present review (which focuses on predictive modeling mainly using clinical data) and deserves the undivided attention of the research community [83-85]. However we did find two instances where DL was applied within the scope of the present review[86,87]. Another family of models that consistently perform well in cancer outcomes prediction is the one comprising different forms of ensembles: i.e. instances where a set of models is trained, instead of a single one, and their output is combined in various ways in order to provide the final prediction. Some of the most notable are approaches using bagging like random forests $(\mathrm{RF})[75,86,88]$, approaches based on boosting (e.g. XGB or gradient boosted decision trees)[73,89,90] and voting ensembles[71,75]. A rather original approach is employed by Morino [91] who used temporal expert advice (TEA) where a set of "experts", each represented by another patients' time series, were combined in a weighted voting scheme. Despite the good performance that more complex ML algorithms provide, it is also worth mentioning the fact that simpler models also bring value, especially when the predictive model has to be deployed in the clinical setting to assist decision making. Complex models indeed quickly lose appeal when interpretability, and ease of use come into the picture. Models where a limited set of features is used as input (e.g. and selection is performed through features selection) may favor implementation in web [78,92] and mobile [75] tools that are ready to use at the bedside. Finally, feature selection and feature engineering can be effective tools to take advantage of prior knowledge, in order to boost an ML model performance [93,94] complementing a purely data-driven approach with expert knowledge.

\subsubsection{Precision medicine principles help predictive modeling}

Some research efforts about predictive modeling in cancer can significantly benefit from principles promoted by precision medicine, especially considering its strong focus on oncological applications [95]. Subgroup analysis based on patient similarity, clustering based on big data and unsupervised phenotype discovery [96] can all improve cancer outcome prediction.

In the current review, two papers $[69,74]$ highlight how developing stage-specific models, despite the potential reduction in the size of patient-cohorts, proved to be beneficial to performance, while evaluating predictive models for survivability on all the stages together might lead to systematically overestimating their predictive performance.

As in the above example, some studies found evidence that being able to stratify patients before the actual modeling is beneficial. Kawakami et al. [89] advocate that use of predictive algorithms 
(in particular a combination of supervised and unsupervised approaches) may facilitate personalizing treatment options through pretreatment stratification of patients. Another example of successful application of unsupervised ML algorithms can be found in Lynch et al. [73], who used unsupervised data analysis techniques like self-ordering maps and k-Means to classify patients by defining the classes as effective proxies for survival prediction. Their study on lung cancer patients from SEER suggests comparable results to state-of-the-art supervised regression techniques, such as gradient-boosting machine. Despite some evidence of their potential usefulness, we report an under-utilization of unsupervised ML approaches in the analyzed articles.

\subsection{Coaching}

Given improved cancer treatments and increasing survival rates, there arises a need to manage HRQoL of cancer patients [97]. Evidence suggests that HRQoL is a significant prognostic predictor, where lower levels are associated with poorer survival, yet it is affected by a number of disease- and treatment-related side effects. Multiple attempts of addressing these side effects with digital interventions implemented as mobile or web-based apps have been described in the literature, and in this section we review selected works. In Section 3.4.1 we focus on different aspects of patient behavior that correspond to specific HRQoL dimensions. Moreover, in Section 3.4.2 we present behavior change techniques and other theoretical models employed by the reviewed systems.

\subsubsection{Addressed aspects of patient behavior}

The prevalent aspect of patient behavior is physical activity (PA) that is discussed in [98-101]. Reviewed studies involve both generic apps developed for general audiences, as well as specialized apps aimed specifically at cancer patients. Use of generic apps and activity trackers for encouraging and monitoring physical activity in breast, prostate and colorectal cancer patients was described in and [98] and [99]. While these interventions resulted in increased physical activity and better awareness of lifestyle (e.g, through idle alerts), the patients pointed at several shortcomings. The major one was the lack of customization for specific patients that would take into account limitations imposed by their disease and treatment. Such customization should for example cover the type of suggested activities with walking being considered as the easiest and safest one, and the frequency and tone of reminders. Other shortcomings included inability to define goals for such activities as swimming or cycling and to automatically track them. Moreover, patients suggested such interventions should be prescribed as a routine part of care and there should be a possibility of discussing them with a healthcare provider, e.g., a nurse specialist.

A dedicated web-based system - RiseTx - for reducing sedentary behavior in prostate cancer patients was described by Trinh et al. [100]. RiseTx offers a multi-phase intervention that involves self-monitoring to establish baseline, action planning and progressive release of selfregulatory strategies, consolidation and maintenance. The system also uses incentives to increase engagement -- patients obtain reward points that they can redeem for various items or donate to charity. In the feasibility study the authors observed significant decrease of sedentary time at post-intervention, but this change was lost at follow-up which suggests challenges with sustaining engagement once the intervention had been completed. Another specialized app 
targeting PA is BENECA presented in [101] and implemented as a mobile app. BENECA supports cancer patients in managing their energy by monitoring its intake (diet) and expenditure (PA) and by providing immediate feedback and recommendations based on guidelines and systematic reviews. The system was verified in 8-week study - while its use posed some challenges with collecting diet-related data, it improved several QoL scores (captured with EORTC QLQ-C30), e.g., global health, physical functioning or cognitive functioning. Moreover, its users felt more motivated to increase the level of PA.

Other frequently discussed aspects of behavior are related to mental well-being and include depression [102], distress and anxiety [1,2]. In [102] Chow et al. present the iCanThrive mobile app that helps women's cancer survivors reduce symptoms of depression. The app offers 8 modules with interactive exercises, e.g., challenging a negative thought. In a 6-week pilot study the authors observed a significant reduction in symptoms of depression at post-intervention. Moreover, there was no significant difference between post-intervention and follow-up. Smith et al. in [103] described application of the PTSD Coach mobile app developed by the US Department of Veteran Affairs to manage post-traumatic stress disorder in cancer patients. The app provides educational resources, self-assessment tools extended with interpretative feedback and a set of mind-body exercises. PTSD Coach was tested in an 8-week pilot study where most of the participants were satisfied with the app and nearly half of them reported improvement in their symptoms. Finally, Kubo et al. [104] described the use of the Headspace app to improve mental well-being of cancer patients undergoing chemotherapy and their caregivers. Results of an 8-week study showed benefits in terms of statistically significant reduction of distress, anxiety and depression. It also revealed several interesting findings -- the baseline level of distress and anxiety was higher for caregivers than for patients, and patients who participated with their caregivers demonstrated better adherence with the app and experienced stronger reduction in depression.

The last group of papers focuses on efficacy (improved skills) for handling problems associated with disease and its symptoms [105,106]. Wklander et al. [105] describe the web app developed as part of the Fex-Can (Fertility and Sexuality Following Cancer) project aimed at adolescent and young adults. The app supports the patients in improving their skills to target sexual and fertility-related problems by delivering a mixed educational content including articles, exercises and video vignettes (real stories patients can relate to). The app underwent a 2-month feasibility study where more than half participants remained active throughout that period and considered the content to be relevant and informative. Finally, Beck et al. [106] describe the SymptomCare@Home system focused on patients receiving chemotherapy. The system facilitates reporting of relevant symptoms through IVR (interactive voice response), it also delivers immediate feedback and self-management coaching appropriate for the patient's state and treatment toxicity. The coaching is evidence-based -- it builds on clinical guidelines and literature and covers a whole range of topics, such as preventing weight gain, improving eating, improving concentration and thinking and relieving pain. The system underwent a randomized cliinical trial where its users had significantly less overall symptom severity and reduction in the number of days with moderate and severe symptoms. 


\subsubsection{Applied behavior change techniques and other models}

Authors of selected studies also discussed behavior change techniques (BCTs) and other theoretical constructs employed in considered apps. First, Kalke et al. [107] reviewed 30 apps for breast cancer patients available to the general public in popular app stores and covering nearly all cancer continuum with the exception of end-of-life support. The authors used the taxonomy for coding BCTs in mobile cancer apps [108] (this taxonomy is based on the original proposal by Abraham and Michie [109]) and identified 12 BCTs coming from 6 categories: customization, information/behavior relationship, intention, facilitation, self-efficacy and social influence. BCTs from the first four categories were the most prevalent ones -- they were found in at least half of the reviewed apps. Moreover, BCTs aimed at facilitation (i.e., providing instructions, materials and education) were implemented in $80 \%$ of the apps. The authors also found out that the apps with user star ratings offered significantly more BCTs than these without ratings.

Similarly, Roberts et al. [99] identified BCTs in generic PA mobile apps that can be used by cancer patients. They identified the same categories of BCTs and in the previous study with the exception of customization, however, this difference could be attributed to using the original taxonomy by Abraham and Michie that does not explicitly mention the customization and personalization category. BCTs were also mentioned in the context of the RiseTx system [100] where in addition to intention-related BCTs as action and coping planning the authors also employed contingent awards (incentives) to increase engagement with the intervention. Finally, the BENECA system [101] employed BCTs from the intention, facilitation and self-efficacy categories.

Finally, the authors of the iCanThrive app [102] employed the efficiency model of support that identifies failures that prevent users from benefiting from a digital intervention, including issues related to the usability of the app, its fit to the user's needs, knowledge on how to use the app and implementation failures. The model was used to develop protocols supporting phone calls aimed at improving engagement with the app, promoting knowledge of the skills found in the app and encouraging implementation of these skills in daily life.

\section{Discussion}

Our analysis of the papers identified dimensions that emerged and were common to papers from the four scope areas. Table 3 summarizes the how papers in the four scope areas addressed the dimensions of cancer type, cancer stage, the evaluation done, and year of publication.

Table 3. Common metadata about the papers reviewed. Cancer types have been ordered top-tobottom according to their incidence (incidence source data [102]).

\begin{tabular}{|l|l|l|l|l|l|}
\hline Scope area & $\begin{array}{l}\text { Data } \\
\text { Collection } \\
(50 \text { papers })\end{array}$ & $\begin{array}{l}\text { Data } \\
\text { Integration } \\
(13 \text { papers })\end{array}$ & $\begin{array}{l}\text { Predictive } \\
\text { modeling } \\
(36 \text { papers })\end{array}$ & $\begin{array}{l}\text { Coaching } \\
(10 \text { papers })\end{array}$ \\
\hline
\end{tabular}




\begin{tabular}{|c|c|c|c|c|c|}
\hline Dimension & & & & & \\
\hline \multirow[t]{9}{*}{ Cancer type } & Lung & $\begin{array}{l}3 \\
{[35,110,111} \\
]\end{array}$ & $1[59]$ & $\begin{array}{l}6 \\
{[64,66,68,82,} \\
83,112]\end{array}$ & \\
\hline & Breast & \begin{tabular}{|l}
5 \\
{$[24,25,27,3$} \\
$8,113]$
\end{tabular} & $\begin{array}{l}5 \\
{[53,55,57,64,} \\
67]\end{array}$ & $\begin{array}{l}5 \\
{[69,72,90,114} \\
, 115]\end{array}$ & \begin{tabular}{|l}
8 \\
{$[72,98,99,101$} \\
$-105]$
\end{tabular} \\
\hline & Colorectal & $2[22,47]$ & $\begin{array}{l}4 \\
{[53,57,61,68]}\end{array}$ & \begin{tabular}{|l}
6 \\
{$[26,76,77,90$} \\
$116,117]$
\end{tabular} & $1[99]$ \\
\hline & Prostate & $2[18,118]$ & & $\begin{array}{l}5[86,89,119- \\
121]\end{array}$ & $3[93,95,98]$ \\
\hline & Head \& Neck & \begin{tabular}{|l}
5 \\
{$[29,33,111$,} \\
$112,122]$
\end{tabular} & $1[56]$ & \begin{tabular}{|l}
5 \\
{$[78,79,118,11$} \\
$9,123]$
\end{tabular} & \\
\hline & Gynaecologic & $\begin{array}{l}3 \\
{[20,41,124]}\end{array}$ & $1[50]$ & $3[81,84,123]$ & $1[97]$ \\
\hline & Bladder & $1[105]$ & & $1[106]$ & \\
\hline & Hematologic & & & & 3 [103-105] \\
\hline & $\begin{array}{l}\text { Other / not } \\
\text { specified }\end{array}$ & $\begin{array}{l}29 \\
{[20,26,28,3} \\
0- \\
32,34,37,39 \\
40,43,45,4 \\
6,49,110,12 \\
0,121,124- \\
135]\end{array}$ & $4[60,62-64]$ & \begin{tabular}{|l}
5 \\
{$[69,92,93,136$} \\
$, 137]$
\end{tabular} & 3 [104-107] \\
\hline \multirow[t]{2}{*}{ Cancer stage } & Diagnosis & & $1[55]$ & & \\
\hline & Treatment & $\begin{array}{l}33 \\
{[21,21,25,2} \\
6,28,29,34, \\
39- \\
43,46,49,52 \\
110,111,11 \\
3,120- \\
122,124,12 \\
5,127-\end{array}$ & 1 [54] & $\begin{array}{l}7 \\
{[78,91,93,114} \\
, 119,137,139]\end{array}$ & $\begin{array}{l}3 \\
{[100,104,106]}\end{array}$ \\
\hline
\end{tabular}




\begin{tabular}{|c|c|c|c|c|c|}
\hline & & $135,138]$ & & & \\
\hline & Post-treatment & $\begin{array}{l}4 \\
{[20,23,47,1} \\
12]\end{array}$ & $1[64]$ & $\begin{array}{l}5 \\
{[79,88,90,94,} \\
140]\end{array}$ & $\begin{array}{l}4 \\
{[27,98,99,102} \\
]\end{array}$ \\
\hline & $\begin{array}{l}\text { Both treatment } \\
\text { and post- } \\
\text { treatment }\end{array}$ & $\begin{array}{l}3 \\
{[33,45,50]}\end{array}$ & $1[57]$ & $\begin{array}{l}9 \\
{[70,76,87,89,} \\
92,116,118,1 \\
23,141]\end{array}$ & \\
\hline & Palliative & $\begin{array}{l}4 \\
{[22,30,32,3} \\
7]\end{array}$ & & & \\
\hline & $\begin{array}{l}\text { Other / not } \\
\text { specified }\end{array}$ & $\begin{array}{l}6 \\
{[24,27,31,3} \\
5,38,126]\end{array}$ & $\begin{array}{l}5 \\
{[54,61,64,67,} \\
68]\end{array}$ & $\begin{array}{l}15[69,70,72- \\
76,78,87,111, \\
115,137,142- \\
144]\end{array}$ & $\begin{array}{l}3 \\
{[104,106,108]}\end{array}$ \\
\hline \multirow[t]{2}{*}{$\begin{array}{l}\text { Evaluation } \\
\text { done }\end{array}$} & $\begin{array}{l}\text { Data set size } \\
\text { used for } \\
\text { evaluation of } \\
\text { integration: } \\
\text { Small }(<100 \\
\text { patients, }<50 \\
\text { elements): }\end{array}$ & $\begin{array}{l}\text { Small: } 27 \\
{[21,22,24-} \\
26,28,30,31 \\
34,35,37,4 \\
1,43,47- \\
49,51,112,1 \\
14,121,123, \\
124,127,13 \\
5,136,136,1 \\
38,139]\end{array}$ & Small: 1 [54] & $\begin{array}{l}\text { Applicable } \\
\text { only to } \\
\text { studies } \\
\text { reporting } \\
\text { external } \\
\text { validation } \\
\text { Small: } 3 \\
{[79,80,145]}\end{array}$ & $\begin{array}{l}\text { Small: } 8 \text { [99- } \\
\text { 106] }\end{array}$ \\
\hline & $\begin{array}{l}\text { Medium } \\
(<1000 \\
\text { patients, <100 } \\
\text { elements): } \\
\text { Large: }\end{array}$ & $\begin{array}{l}\text { Medium: } 7 \\
{[23,29,39,4} \\
0,44,46,110 \\
] \\
\text { Large: } 3 \\
{[32,131,132} \\
]\end{array}$ & $\begin{array}{l}\text { Medium: } 5 \\
{[55,56,58,61 \text {, }} \\
66] \\
\text { Large: } 4 \\
{[57,63,66,67]}\end{array}$ & & $\begin{array}{l}\text { Medium: } 1 \\
{[107]}\end{array}$ \\
\hline
\end{tabular}




\begin{tabular}{|l|l|l|l|l|l|}
\hline Publication & 2015 & 2 & 1 & 2 & 0 \\
year & 2016 & 3 & 5 & 4 & 0 \\
& 2017 & 7 & 0 & 9 & 2 \\
& 2018 & 5 & 2 & 4 & 3 \\
& 2019 & 17 & 5 & 15 & 3 \\
& 2020 & 16 & 0 & 2 & 2 \\
\hline
\end{tabular}

The above Table 3 allows some interesting observations. First, Breast cancer is the most represented in the group of papers we analyzed. Neither melanoma or kidney cancer, which are the focus of the CAPABLE research project, have been explicitly addressed in any of the retrieved articles. Number of articles focusing on a certain cancer type are correlated to different cancer types incidence. The topic of our review that covered the least prevalent types of cancers is coaching. However lung and head\&neck cancers had no coaching system explicitly addressing them, probably because of their short course and limited survival.

Secondly, we chose to only focus on already diagnosed cancer patients. For this reason Table 3 reports cancer stages other than diagnosis, thus starting from the treatment stage. What is more, cancer stage did not make a difference for works about data integration. Finally, the palliative stage is generally under-supported, and indeed no data integration, predictive modeling or coaching paper addressed it. However, it is worth mentioning that, especially for coaching systems, not only patients but also home caregivers are likely to benefit from support in this endof-life stage.

For what regards size of the evaluation, most papers $37 / 50$ of the data collection area performed some evaluation: most of them (27) on a small sample size, and less (7 and 3) on a medium or large sample. On the other hand, only three articles on predictive modeling performed external validation on small sets of patients. Most (9/10) coaching system papers performed evaluation, albeit on small cohorts.

Finally, a trend for a generalized growing interest in all data science areas can be pointed out from Table 3 data. In particular, electronic data collection and predictive modeling are becoming more popular in the last 2 years.

\subsection{Best-practices and open research challenges}

We present the best practices and open challenges that we identified in each scope area.

\subsubsection{Data Collection Best Practices}

We identified the following best practices regarding data collection:

a. Use validated questionnaires to collect PROs

b. Choose a set of PRO questionnaires that covers multiple aspects of wellbeing, including physical, mental, social, activities and functioning, and symptoms. 
c. EORTC QLQ-C30 has good coverage of well-being dimensions and is validated. It is the most widely used PRO questionnaire in Europe (used in 14 of the 50 studies reviewed in the area of data collection). Specific cancer versions are available or currently under validation (e.g. QLQ-MEL38)

d. Collect outcome data at least at baseline and after the intervention, but preferably during the intervention as well.

e. Presently, sensors offered by smartphones are not a reliable and accurate source of measurable data.

\subsubsection{Data Collection Open Challenges}

i. Develop easy methods for collecting PROs and symptoms from recovering patients and survivors in their home environments, while they are undergoing the intervention. This could be facilitated by the two methods below.

ii. Develop computer-adapted testing versions of validated PRO questionnaires that have over twenty questions in order to make it easier for patients to complete them.

iii. Reliable and easy to use mobile/wearable sensors could be used to collect vital signs, symptoms and PRO data (e.g., activity and functioning) when possible, relieving patients from the burden of self-reporting of symptoms and from the bias of reporting untruthful data. The degree of correlation between monitors routinely used in clinical practice and the smartphone-based applications is insufficient to recommend clinical utilization of the latter. This lack of correlation suggests that the smartphones-based applications do not provide clinically meaningful data. The inaccurate data provided by these applications can potentially contribute to patient harm.

\subsubsection{Data Integration Best Practices}

We identified the following best practices regarding data integration:

a. Semantic data integration makes the data set more comprehensive -- larger data sets or data sets that include more data items about the patients. This improves the ability to infer more valid results from data analysis.

b. Data integration should include extract, transform, load (ETL) functions that allow pulling data from a source database (e.g., hospital information system) and storing it in a target database.

c. The ETL process can benefit from basing the target database on the Observational Medical Outcomes Partnership (OMOP) common data model (CDM), as in KETOS [53]. OMOP provides services (based on $\mathrm{R}$ ) to perform distributed analysis of data based on the OMOP semantics. It will help researchers to compare their data and results. Observational Health Data Sciences and Informatics (OHDSI), which is the organization that develops OMOP, focuses on horizontal partitioning of data sets with similar attributes. Projects that span multiple sites, such as CAPABLE, can benefit from this platform for distributed analysis.

d. Data should meet principles of findability, accessibility, interoperability, and reusability (FAIR). OMOP provides the basis for Interoperability of the data. Findability and Accessibility are facilitated by depositing metadata in a repository (e.g., Zenodo) that 
describes the data and the access conditions. Reusability is realized by clear and as-openas-possible licensing, targeting at CC-BY (which allows others to distribute in altered or unaltered form, also commercially, as long as credit is given) and CC-0 (i.e., "No Rights Reserved").

e. The most popular patient information model used was FHIR. It is a standard used by commercial vendors for health information exchange. Supporting it will ease data exchange and potential commercialization.

f. Standard vocabularies (ICD, LOINC, SNOMED CT and RxNorm) are used by most of the papers to capture the foci of FHIR or OMOP objects.

g. The ETL process can be done manually in a process by which local sites define mappings between source data elements (SDE) to a set of needed common data elements (CDE). The manual process could best be supported by terminology services to locate standard terms and their meaning.

h. The integration process may also be supported by the use of machine learning, either to harmonize data, or to perform analyses on pluriform data that has not been harmonized.

\subsubsection{Data Integration Open Challenges}

i. Best-practice ETL processes benefit by exporting data from EHRs or data warehouses into an OMOP common data model database. However, OMOP does not provide a data exchange/communication standard. We recommend adding a FHIR API layer to allow components of a complex personalized knowledge-based and prediction-based decisionsupport system to semantically share data and knowledge through a standardized API. Combining OMOP, FHIR, and controlled terminologies for the ETL target database, leverages the benefits of all of these standards resulting in a "best of breed" approach for storage and exchange.

ii. The papers reviewed above do not address the semantic gap that exists between formalized clinical knowledge used for decision support and raw data stored in integrated ETL target databases. The knowledge sources, such as computer-interpretable clinical guidelines [144], contain abstract terms (e.g., complicated diarrhea) that can be computed from raw data about symptoms that are stored in the database. A component such as Knowledge-Data Ontology Mapper [146] or the Medical Database Adaptor (MEIDA) [147] could be added to bridge the semantic gap by allowing modelers to provide ontological definitions of abstractions in terms of a standard patient data model and based on it, automatic query generation. However, both of these mediators have shortcomings, described in [146], and should be extended to support the full range of abstractions needed for decision support.

\subsubsection{Predictive modeling Best Practices}

We identified the following best practices regarding predictive modeling:

a. Some articles deal with unbalanced data and cope with it mostly with undersampling. 
b. Stratifying patients in subgroups before predictive modeling promotes better predictive performances.

\subsubsection{Predictive modeling Open Challenges}

i. $\quad$ Predictive models should be validated on unseen patient datasets. Most studies do not perform external validation, but instead rely on more conventional cross validation or train-test splits, which tend to overestimate performance [142,143].

ii. None of the predictive modeling papers have considered using PRO data as predictors of good/bad outcome or survival. The validity of AI-based inference of patients' psychobehavioral dimensions [148] (e.g., stress, anxiety, sleep quality, depression) from PRO and sensor data needs to be assessed.

iii. Unsupervised approaches are underutilized for prediction of a patient's state based on clinical, PRO and sensor data.

\subsubsection{Coaching Systems Best Practices}

We identified the following best practices regarding patient coaching .

a. Behavioral interventions (e.g., physical exercise) for cancer patients offered by apps should be suited for the characteristics of cancer patients rather than be general-purpose. Robertson et al. [149] presented a hypothetical app that provided interesting suggestions given by cancer patients which should be considered:

- Messages to patients should use a casual (not clinical), concise, and positive tone. This is in line with recommendations from the IOM on the language that should be used in mHealth apps [150]

- The app should include tools that support personal goal attainment (e.g., reminders, role model narratives)

- Value-based goals should be included (e.g., "I want to see my grandchildren married"). They should be attained by short-terms goals (e.g., for physical activity) that are easy to attain.

- Recommendations should come from a trusted source, and should include layman summary of the relevant literature justifying the recommendation

- Recommendations should preferably include video demonstrations, preferred over text and pictures. Exercises could be presented by other cancer patients.

- Interventions should deliver an experience that is tailored to the user's characteristics (cancer-related information, age,...), also taking into account the location of the patient and weather

- Whenever possible, wearables should be used to track patients; this lowers the burden of data entry.

- Adherence should be rewarded (e.g., by congratulations messages), yet competition (e.g., leader boards) was not well received; patients preferred more private social experience, with a small group of friends and family [151].

- Novel ways that provide patients with social support via the app should be developed and validated. Social support is not included in most existing apps. 
b. Ginossar et al. presented a set of recommendations related to the usability of mHealth apps for breast cancer patients and the language used in these apps [150] that are applicable to other types of cancer:

i. Usability recommendations include easy access to home page and clearly labeled back button, in-app simple search and browsing of provided content and use of images to facilitate learning

ii. Language recommendations include using the pronoun "you", present tense, active voice and action words, limiting the length of sentences and paragraphs, and using common everyday words and defining technical terms

c. According to Prochaska et al. [148] participation in content creation results in a stronger intention for behavior change (e.g. quitting smoking). Moreover, posting photos is associated with stronger engagement than for other types of posted materials.

It is interesting to note that the aspects of behavior most frequently considered when providing coaching include physical activity/sedentary behavior (also combined with diet), distress and anxiety, and self-efficacy.

\subsubsection{Coaching Systems Open Challenges}

i. App design should rely on sound behavioral theories or models. Many available apps are intuitive and use formally defined digital interventions (e.g., captured by the initial taxonomy by Abraham and Michie [109] and by taxonomies revised for cancer apps [108]), and the frequently used categories of digital interventions involve: customization, information/behavior relationship, intention, facilitation, self-efficacy and social influence. However, identification of applied interventions is often performed only during post-hoc analysis, instead of being part of the design process.

ii. A controlled study should be used to measure improvement in QoL. Yet attributing improvement to particular system functionality necessitates large cohorts partitioned into groups receiving different app versions or a study in which different features are exposed over time, or even several studies (e.g., as for the CHESS system where different trials were conducted to assess the impact of several combinations of patient-oriented support [152]).

iii. Coaching systems for the palliative stage could potentially be developed; none of the papers in our review addressed this important stage, although home caregivers are likely to benefit from such systems.

\subsection{Limitations}

Our review study presents some limitations. First of all, the results presented in Section 3, and the discussion of best-practices and open research challenges presented in Section 4.1 are based solely on our particular set of papers reviewed.

A second limitation is that we did not include "well-being", an important construct in cancer outcomes research, as part of our search keywords. However this was mitigated by the fact that the multidimensionality of QoL refers to a broad range of content, including the concepts of well-being [18]. 


\section{Conclusion}

We reported results of a systematic literature review about the state-of-the-art of computerized systems that employ Data Science methods to monitor the health status and provide support to cancer patients. Our analysis identified best practices that we intend to adopt in the CAPABLE project. In addition, we plan to address some of the open research challenges that are not currently addressed. These include supporting emotional and social dimensions of well-being, unobtrusive monitoring through wearable sensors, including PROs in predictive modeling and providing better customization of behavioral interventions for the specific population of cancer patients.

\section{Acknowledgements}

The work described in this article has been funded by the European Union's Horizon 2020 research and innovation programme under grant agreement No 875052 - CAPABLE (www.capable-project.eu).

\section{References}

[1] Greenes RA, editor. Clinical Decision Support: the road to a broad adoption. Oxford: Academic Press; 2014.

[2] Riaño D, Peleg M, ten Teije A. Ten years of knowledge representation for health care (2009-2018): Topics, trends, and challenges. Artificial Intelligence in Medicine 2019;100:101713. https://doi.org/10.1016/j.artmed.2019.101713.

[3] Elsevier. AIIM aims and scope n.d. https://www.sciencedirect.com/journal/artificialintelligence-in-medicine.

[4] Wing JM. The Data Life Cycle. Harvard Data Science Review 2019. https://doi.org/10.1162/99608f92.e26845b4.

[5] Donoho D. 50 Years of Data Science. Journal of Computational and Graphical Statistics 2017;26:745-66. https://doi.org/10.1080/10618600.2017.1384734.

[6] Aerts HJWL. Data Science in Radiology: A Path Forward. Clin Cancer Res 2018;24:532-4. https://doi.org/10.1158/1078-0432.CCR-17-2804.

[7] Vedula SS, Hager GD. Surgical data science: the new knowledge domain. Innov Surg Sci 2017;2:109-21. https://doi.org/10.1515/iss-2017-0004.

[8] Gruson D, Helleputte T, Rousseau P, Gruson D. Data science, artificial intelligence, and machine learning: Opportunities for laboratory medicine and the value of positive regulation. Clinical Biochemistry 2019;69:1-7. https://doi.org/10.1016/j.clinbiochem.2019.04.013.

[9] Kotz D, Lord SE, O’Malley AJ, Stark L, Marsch LA. Workshop on Emerging Technology and Data Analytics for Behavioral Health. JMIR Res Protoc 2018;7:e158. https://doi.org/10.2196/resprot.9589.

[10] cancer.org. Managing Cancer as a Chronic Illness. CancerOrg n.d. https://www.cancer.org/treatment/survivorship-during-and-after-treatment/when-cancerdoesnt-go-away.html (accessed July 16, 2020).

[11] Donoho D. 50 Years of Data Science. Journal of Computational and Graphical Statistics 2017;26:745-66. https://doi.org/10.1080/10618600.2017.1384734. 
[12] Moher D, Liberati A, Tetzlaff J, Altman DG, Group TP. Preferred Reporting Items for Systematic Reviews and Meta-Analyses: The PRISMA Statement. PLOS Medicine 2009;6:e1000097. https://doi.org/10.1371/journal.pmed.1000097.

[13] Text Words [tw] n.d. https://www.nlm.nih.gov/bsd/disted/pubmedtutorial/020_760.html (accessed April 20, 2020).

[14] Gates A. Technology-assisted title and abstract screening for systematic reviews: a retrospective evaluation of the Abstrackr machine learning tool 2018:9.

[15] Critical Appraisal Skills Programme. CASP qualitative Checklist 2019. https://caspuk.net/wp-content/uploads/2018/01/CASP-Qualitative-Checklist-2018.pdf (accessed December 23, 2020).

[16] Linton M-J, Dieppe P, Medina-Lara A. Review of 99 self-report measures for assessing well-being in adults: exploring dimensions of well-being and developments over time. BMJ Open 2016;6:e010641. https://doi.org/10.1136/bmjopen-2015-010641.

[17] Cella DF. Quality of life: Concepts and definition. Journal of Pain and Symptom Management 1994;9:186-92. https://doi.org/10.1016/0885-3924(94)90129-5.

[18] EORTC Quality of Life website | EORTC Quality of Life Group website. EORTC Quality of Life n.d. https://qol.eortc.org/ (accessed June 30, 2020).

[19] Hambleton RK, Swaminathan H, Rogers HJ. Fundamentals of Item Response Theory. SAGE; 1991.

[20] Avery KNL, Richards HS, Portal A, Reed T, Harding R, Carter R, et al. Developing a realtime electronic symptom monitoring system for patients after discharge following cancerrelated surgery. BMC Cancer 2019;19:463. https://doi.org/10.1186/s12885-019-5657-6.

[21] Sundberg K, Wengström Y, Blomberg K, Hälleberg-Nyman M, Frank C, Langius-Eklöf A. Early detection and management of symptoms using an interactive smartphone application (Interaktor) during radiotherapy for prostate cancer. Support Care Cancer 2017;25:2195204. https://doi.org/10.1007/s00520-017-3625-8.

[22] Soh JY, Cha WC, Chang DK, Hwang JH, Kim K, Rha M, et al. Development and Validation of a Multidisciplinary Mobile Care System for Patients With Advanced Gastrointestinal Cancer: Interventional Observation Study. JMIR Mhealth Uhealth 2018;6:e115. https://doi.org/10.2196/mhealth.9363.

[23] Cowan RA, Suidan RS, Andikyan V, Rezk YA, Einstein MH, Chang K, et al. Electronic patient-reported outcomes from home in patients recovering from major gynecologic cancer surgery: A prospective study measuring symptoms and health-related quality of life. Gynecol Oncol 2016;143:362-6. https://doi.org/10.1016/j.ygyno.2016.08.335.

[24] Lozano-Lozano M, Melguizo-Rodríguez L, Fernández-Lao C, Galiano-Castillo N, Cantarero-Villanueva I, Martín-Martín L, et al. Association Between the Use of a Mobile Health Strategy App and Biological Changes in Breast Cancer Survivors: Prospective PrePost Study. J Med Internet Res 2019;21:e15062. https://doi.org/10.2196/15062.

[25] Kikawa Y, Hatachi Y, Rumpold G, Tokiwa M, Takebe S, Ogata T, et al. Evaluation of health-related quality of life via the Computer-Based Health Evaluation System (CHES) for Japanese metastatic breast cancer patients: a single-center pilot study. Breast Cancer 2019;26:255-9. https://doi.org/10.1007/s12282-018-0905-1.

[26] Abdur Rahman M, Rashid MM, Le Kernec J, Philippe B, Barnes SJ, Fioranelli F, et al. A Secure Occupational Therapy Framework for Monitoring Cancer Patients' Quality of Life. Sensors (Basel) 2019;19. https://doi.org/10.3390/s19235258. 
[27] Lozano-Lozano M, Martín-Martín L, Galiano-Castillo N, Fernández-Lao C, CantareroVillanueva I, López-Barajas IB, et al. Mobile health and supervised rehabilitation versus mobile health alone in breast cancer survivors: Randomized controlled trial. Ann Phys Rehabil Med 2019. https://doi.org/10.1016/j.rehab.2019.07.007.

[28] Adams JR, Ray D, Willmon R, Pulgar S, Dasari A. Living With Neuroendocrine Tumors: Assessment of Quality of Life Through a Mobile Application. JCO Clin Cancer Inform 2019;3:1-10. https://doi.org/10.1200/CCI.19.00025.

[29] Van Cleave JH, Fu MR, Bennett AV, Persky MS, Li Z, Jacobson A, et al. The development, usability, and reliability of the Electronic Patient Visit Assessment (ePVA) for head and neck cancer. Mhealth 2019;5:21. https://doi.org/10.21037/mhealth.2019.06.05.

[30] Buergy D, Siefert V, Neumaier C, Ganslandt T, Sperk E, Blessing M, et al. Prospective trial on telemonitoring of geriatric cancer patients using handheld devices. Strahlenther Onkol 2020;196:205-12. https://doi.org/10.1007/s00066-019-01548-0.

[31] van der Hout A, van Uden-Kraan CF, Holtmaat K, Jansen F, Lissenberg-Witte BI, Nieuwenhuijzen GAP, et al. Role of eHealth application Oncokompas in supporting selfmanagement of symptoms and health-related quality of life in cancer survivors: a randomised, controlled trial. Lancet Oncol 2020;21:80-94. https://doi.org/10.1016/S14702045(19)30675-8.

[32] Pavic M, Klaas V, Theile G, Kraft J, Tröster G, Blum D, et al. Mobile Health Technologies for Continuous Monitoring of Cancer Patients in Palliative Care Aiming to Predict Health Status Deterioration: A Feasibility Study. J Palliat Med 2019. https://doi.org/10.1089/jpm.2019.0342.

[33] Zini EM, Lanzola G, Quaglini S, Bossi P, Licitra L, Resteghini C. A pilot study of a smartphone-based monitoring intervention on head and neck cancer patients undergoing concurrent chemo-radiotherapy. Int J Med Inform 2019;129:404-12. https://doi.org/10.1016/j.ijmedinf.2019.06.004.

[34] Wang J, Yao NA, Liu Y, Geng Z, Wang Y, Shen N, et al. Development of a Smartphone Application to Monitor Pediatric Patient-Reported Outcomes. Comput Inform Nurs 2017;35:590-8. https://doi.org/10.1097/CIN.0000000000000357.

[35] Wagner LI, Schink J, Bass M, Patel S, Diaz MV, Rothrock N, et al. Bringing PROMIS to practice: brief and precise symptom screening in ambulatory cancer care. Cancer 2015;121:927-34. https://doi.org/10.1002/cncr.29104.

[36] Barras D, Missiaglia E, Wirapati P, Sieber OM, Jorissen RN, Love C, et al. BRAF V600E Mutant Colorectal Cancer Subtypes Based on Gene Expression. Clin Cancer Res 2017;23:104-15. https://doi.org/10.1158/1078-0432.CCR-16-0140.

[37] Garcia SF, Wortman K, Cella D, Wagner LI, Bass M, Kircher S, et al. Implementing electronic health record-integrated screening of patient-reported symptoms and supportive care needs in a comprehensive cancer center. Cancer 2019;125:4059-68. https://doi.org/10.1002/cncr.32172.

[38] Nápoles AM, Santoyo-Olsson J, Chacón L, Stewart AL, Dixit N, Ortiz C. Feasibility of a Mobile Phone App and Telephone Coaching Survivorship Care Planning Program Among Spanish-Speaking Breast Cancer Survivors. JMIR Cancer 2019;5:e13543. https://doi.org/10.2196/13543.

[39] Kolb NA, Smith AG, Singleton JR, Beck SL, Howard D, Dittus K, et al. Chemotherapyrelated neuropathic symptom management: a randomized trial of an automated symptom- 
monitoring system paired with nurse practitioner follow-up. Support Care Cancer 2018;26:1607-15. https://doi.org/10.1007/s00520-017-3970-7.

[40] Benze G, Nauck F, Alt-Epping B, Gianni G, Bauknecht T, Ettl J, et al. PROutine: a feasibility study assessing surveillance of electronic patient reported outcomes and adherence via smartphone app in advanced cancer. Ann Palliat Med 2019;8:104-11. https://doi.org/10.21037/apm.2017.07.05.

[41] Graetz I, Anderson JN, McKillop CN, Stepanski EJ, Paladino AJ, Tillmanns TD. Use of a web-based app to improve postoperative outcomes for patients receiving gynecological oncology care: A randomized controlled feasibility trial. Gynecol Oncol 2018;150:311-7. https://doi.org/10.1016/j.ygyno.2018.06.007.

[42] Ciani O, Cucciniello M, Petracca F, Apolone G, Merlini G, Novello S, et al. Lung Cancer App (LuCApp) study protocol: a randomised controlled trial to evaluate a mobile supportive care app for patients with metastatic lung cancer. BMJ Open 2019;9:e025483. https://doi.org/10.1136/bmjopen-2018-025483.

[43] Greer JA, Jacobs JM, Pensak N, Nisotel LE, Fishbein JN, MacDonald JJ, et al. Randomized Trial of a Smartphone Mobile App to Improve Symptoms and Adherence to Oral Therapy for Cancer. J Natl Compr Canc Netw 2020;18:133-41. https://doi.org/10.6004/jnccn.2019.7354.

[44] Modules. EORTC - Quality of Life n.d. https://qol.eortc.org/modules/ (accessed July 30, 2020).

[45] Absolom K, Gibson A, Velikova G. Engaging Patients and Clinicians in Online Reporting of Adverse Effects During Chemotherapy for Cancer: The eRAPID System (Electronic Patient Self-Reporting of Adverse Events: Patient Information and aDvice). Med Care 2019;57 Suppl 5 Suppl 1:S59-65. https://doi.org/10.1097/MLR.0000000000001085.

[46] Galligioni E, Piras EM, Galvagni M, Eccher C, Caramatti S, Zanolli D, et al. Integrating mHealth in Oncology: Experience in the Province of Trento. J Med Internet Res 2015;17:e114. https://doi.org/10.2196/jmir.3743.

[47] Drott J, Fomichov V, Börjeson S, Berterö C. Sense of coherence and health-related quality of life in patients with neurotoxicity after cancer chemotherapy: Assessment from a realtime mobile phone-based system. Psychooncology 2020;29:107-13.

https://doi.org/10.1002/pon.5243.

[48] The PRO-CTCAE Measurement System n.d. https://healthcaredelivery.cancer.gov/proctcae/measurement.html (accessed July 29, 2020).

[49] Sun V, Dumitra S, Ruel N, Lee B, Melstrom L, Melstrom K, et al. Wireless Monitoring Program of Patient-Centered Outcomes and Recovery Before and After Major Abdominal Cancer Surgery. JAMA Surg 2017;152:852-9. https://doi.org/10.1001/jamasurg.2017.1519.

[50] Timmerman JG, Dekker-van Weering MGH, Stuiver MM, Groen WG, Wouters MWJM, Tönis TM, et al. Ambulant monitoring and web-accessible home-based exercise program during outpatient follow-up for resected lung cancer survivors: actual use and feasibility in clinical practice. J Cancer Surviv 2017;11:720-31. https://doi.org/10.1007/s11764-0170611-6.

[51] Chiang A-T, Chen Q, Wang Y, Fu MR. Kinect-Based In-Home Exercise System for Lymphatic Health and Lymphedema Intervention. IEEE J Transl Eng Health Med 2018;6:4100313. https://doi.org/10.1109/JTEHM.2018.2859992. 
[52] Metcalf M, Glazyrine V, Glavin K, Dahlgren A, Michael C, Bechtel M, et al. The Feasibility of a Health Care Application in the Treatment of Patients Undergoing Radical Cystectomy. J Urol 2019;201:902-8. https://doi.org/10.1097/JU.0000000000000050.

[53] Gruendner J, Schwachhofer T, Sippl P, Wolf N, Erpenbeck M, Gulden C, et al. KETOS: Clinical decision support and machine learning as a service - A training and deployment platform based on Docker, OMOP-CDM, and FHIR Web Services. PLoS ONE 2019;14:e0223010. https://doi.org/10.1371/journal.pone.0223010.

[54] Isoviita V-M, Salminen L, Azar J, Lehtonen R, Roering P, Carpén O, et al. Open Source Infrastructure for Health Care Data Integration and Machine Learning Analyses. JCO Clinical Cancer Informatics 2019:1-16. https://doi.org/10.1200/CCI.18.00132.

[55] Mariette J, Villa-Vialaneix N. Unsupervised multiple kernel learning for heterogeneous data integration. Bioinformatics 2018;34:1009-15. https://doi.org/10.1093/bioinformatics/btx682.

[56] Gaebel J, A CM, U LH. Accessing Patient Information for Probabilistic Patient Models Using Existing Standards. Studies in Health Technology and Informatics 2016:107-12. https://doi.org/10.3233/978-1-61499-645-3-107.

[57] Hill-Kayser CE, Jacobs LA, Gabriel P, Palmer SC, Hampshire MK, Vachani C, et al. Feasibility Study of an Electronic Interface Between Internet-Based Survivorship Care Plans and Electronic Medical Records. JOP 2016;12:e380-7. https://doi.org/10.1200/JOP.2015.006841.

[58] Bodenreider O. Biomedical Ontologies in Action: Role in Knowledge Management, Data Integration and Decision Support. Yearb Med Inform 2008;17:67-79. https://doi.org/10.1055/s-0038-1638585.

[59] Longheu A, Carchiolo V, Malgeri M. Medical data integration with SNOMED-CT and HL7. Advances in Intelligent Systems and Computing, 2015. https://doi.org/10.1007/978-3319-16486-1_115.

[60] Warner JL, Jain SK, Levy MA. Integrating cancer genomic data into electronic health records. Genome Med 2016;8:113. https://doi.org/10.1186/s13073-016-0371-3.

[61] Ulrich H, Ann-Kristin K, Petra D-H, K HJ, Josef I. Metadata Repository for Improved Data Sharing and Reuse Based on HL7 FHIR. Studies in Health Technology and Informatics 2016:162-6. https://doi.org/10.3233/978-1-61499-678-1-162.

[62] Dugas M, Meidt A, Neuhaus P, Storck M, Varghese J. ODMedit: uniform semantic annotation for data integration in medicine based on a public metadata repository. BMC Med Res Methodol 2016;16:65. https://doi.org/10.1186/s12874-016-0164-9.

[63] López de Maturana E, Alonso L, Alarcón P, Martín-Antoniano IA, Pineda S, Piorno L, et al. Challenges in the Integration of Omics and Non-Omics Data. Genes (Basel) 2019;10. https://doi.org/10.3390/genes10030238.

[64] Mihaylov I, Kańduła M, Krachunov M, Vassilev D. A novel framework for horizontal and vertical data integration in cancer studies with application to survival time prediction models. Biol Direct 2019;14:22. https://doi.org/10.1186/s13062-019-0249-6.

[65] Warner JL, Patt D, Informatics SE for the IYS on C. Cancer Informatics in 2019: Deep Learning Takes Center Stage. Yearb Med Inform 2020;29:243-6. https://doi.org/10.1055/s0040-1701993.

[66] Firnkorn D, Ganzinger M, Muley T, Thomas M, Knaup P. A Generic Data Harmonization Process for Cross-linked Research and Network Interaction: Construction and Application 
for the Lung Cancer Phenotype Database of the German Center for Lung Research.

Methods Inf Med 2015;54:455-60. https://doi.org/10.3414/ME14-02-0030.

[67] Linkov F, Silverstein J, Davis M, Crocker B, Hao D, Schneider A, et al. Integration of cancer registry data into the text information extraction system: Leveraging the structured data import tool. J Pathol Inform 2018;9:47. https://doi.org/10.4103/jpi.jpi_38_18.

[68] Mate S, Kampf M, Rödle W, Kraus S, Proynova R, Silander K, et al. Pan-European Data Harmonization for Biobanks in ADOPT BBMRI-ERIC. Appl Clin Inform 2019;10:679-92. https://doi.org/10.1055/s-0039-1695793.

[69] Hossein Pour ES, Kate RJ. Stage-Specific Survivability Prediction Models across Different Cancer Types. AMIA Annu Symp Proc 2017;2017:1421-9.

[70] Lynch CM, Abdollahi B, Fuqua JD, de Carlo AR, Bartholomai JA, Balgemann RN, et al. Prediction of lung cancer patient survival via supervised machine learning classification techniques. Int J Med Inform 2017;108:1-8. https://doi.org/10.1016/j.ijmedinf.2017.09.013.

[71] Bergquist SL, Brooks GA, Keating NL, Landrum MB, Rose S. Classifying Lung Cancer Severity with Ensemble Machine Learning in Health Care Claims Data. Proc Mach Learn Res 2017;68:25-38.

[72] Kleinlein R, Riaño D. Persistence of data-driven knowledge to predict breast cancer survival. Int J Med Inform 2019;129:303-11. https://doi.org/10.1016/j.ijmedinf.2019.06.018.

[73] Lynch CM, van Berkel VH, Frieboes HB. Application of unsupervised analysis techniques to lung cancer patient data. PLoS ONE 2017;12:e0184370.

https://doi.org/10.1371/journal.pone.0184370.

[74] Kate RJ, Nadig R. Stage-specific predictive models for breast cancer survivability. Int J Med Inform 2017;97:304-11. https://doi.org/10.1016/j.ijmedinf.2016.11.001.

[75] Oliveira T, Silva A, Satoh K, Julian V, Leão P, Novais P. Survivability Prediction of Colorectal Cancer Patients: A System with Evolving Features for Continuous Improvement. Sensors (Basel) 2018;18. https://doi.org/10.3390/s18092983.

[76] Soguero-Ruiz C, Hindberg K, Rojo-Alvarez JL, Skrovseth SO, Godtliebsen F, Mortensen K, et al. Support Vector Feature Selection for Early Detection of Anastomosis Leakage From Bag-of-Words in Electronic Health Records. IEEE J Biomed Health Inform 2016;20:1404-15. https://doi.org/10.1109/JBHI.2014.2361688.

[77] Soguero-Ruiz C, Hindberg K, Mora-Jiménez I, Rojo-Álvarez JL, Skrøvseth SO, Godtliebsen F, et al. Predicting colorectal surgical complications using heterogeneous clinical data and kernel methods. J Biomed Inform 2016;61:87-96. https://doi.org/10.1016/j.jbi.2016.03.008.

[78] Alabi RO, Elmusrati M, Sawazaki-Calone I, Kowalski LP, Haglund C, Coletta RD, et al. Machine learning application for prediction of locoregional recurrences in early oral tongue cancer: a Web-based prognostic tool. Virchows Arch 2019;475:489-97. https://doi.org/10.1007/s00428-019-02642-5.

[79] Alabi RO, Elmusrati M, Sawazaki-Calone I, Kowalski LP, Haglund C, Coletta RD, et al. Comparison of supervised machine learning classification techniques in prediction of locoregional recurrences in early oral tongue cancer. Int J Med Inform 2019;136:104068. https://doi.org/10.1016/j.ijmedinf.2019.104068.

[80] Bzdok D, Altman N, Krzywinski M. Statistics versus machine learning. Nature Methods 2018;15:233-4. https://doi.org/10.1038/nmeth.4642. 
[81] Beam AL, Kohane IS. Big Data and Machine Learning in Health Care. JAMA 2018;319:1317-8. https://doi.org/10.1001/jama.2017.18391.

[82] Cabitza F, Rasoini R, Gensini GF. Unintended Consequences of Machine Learning in Medicine. JAMA 2017;318:517-8. https://doi.org/10.1001/jama.2017.7797.

[83] Hinton G. Deep Learning-A Technology With the Potential to Transform Health Care. JAMA 2018;320:1101-2. https://doi.org/10.1001/jama.2018.11100.

[84] Wang F, Casalino LP, Khullar D. Deep Learning in Medicine-Promise, Progress, and Challenges. JAMA Intern Med 2019;179:293-4. https://doi.org/10.1001/jamainternmed.2018.7117.

[85] Miotto R, Wang F, Wang S, Jiang X, Dudley JT. Deep learning for healthcare: review, opportunities and challenges. Brief Bioinform 2018;19:1236-46. https://doi.org/10.1093/bib/bbx044.

[86] Matsuo K, Purushotham S, Jiang B, Mandelbaum RS, Takiuchi T, Liu Y, et al. Survival outcome prediction in cervical cancer: Cox models vs deep-learning model. Am J Obstet Gynecol 2019;220:381.e1-381.e14. https://doi.org/10.1016/j.ajog.2018.12.030.

[87] Wang R, Weng Y, Zhou Z, Chen L, Hao H, Wang J. Multi-objective ensemble deep learning using electronic health records to predict outcomes after lung cancer radiotherapy. Phys Med Biol 2019;64:245005. https://doi.org/10.1088/1361-6560/ab555e.

[88] Jochems A, El-Naqa I, Kessler M, Mayo CS, Jolly S, Matuszak M, et al. A prediction model for early death in non-small cell lung cancer patients following curative-intent chemoradiotherapy. Acta Oncol 2018;57:226-30. https://doi.org/10.1080/0284186X.2017.1385842.

[89] Kawakami E, Tabata J, Yanaihara N, Ishikawa T, Koseki K, Iida Y, et al. Application of Artificial Intelligence for Preoperative Diagnostic and Prognostic Prediction in Epithelial Ovarian Cancer Based on Blood Biomarkers. Clin Cancer Res 2019;25:3006-15. https://doi.org/10.1158/1078-0432.CCR-18-3378.

[90] Xu Y, Ju L, Tong J, Zhou C-M, Yang J-J. Machine Learning Algorithms for Predicting the Recurrence of Stage IV Colorectal Cancer After Tumor Resection. Sci Rep 2020;10:2519. https://doi.org/10.1038/s41598-020-59115-y.

[91] Morino K, Hirata Y, Tomioka R, Kashima H, Yamanishi K, Hayashi N, et al. Predicting disease progression from short biomarker series using expert advice algorithm. Sci Rep 2015;5:8953. https://doi.org/10.1038/srep08953.

[92] Ferroni P, Zanzotto FM, Scarpato N, Riondino S, Guadagni F, Roselli M. Validation of a Machine Learning Approach for Venous Thromboembolism Risk Prediction in Oncology. Dis Markers 2017;2017:8781379. https://doi.org/10.1155/2017/8781379.

[93] Mi H, Petitjean C, Dubray B, Vera P, Ruan S. Robust feature selection to predict tumor treatment outcome. Artif Intell Med 2015;64:195-204. https://doi.org/10.1016/j.artmed.2015.07.002.

[94] Lin Y-T, Lee MT-S, Huang Y-C, Liu C-K, Li Y-T, Chen M. Prediction of Recurrenceassociated Death from Localized Prostate Cancer with a Charlson Comorbidity Indexreinforced Machine Learning Model. Open Med (Wars) 2019;14:593-606. https://doi.org/10.1515/med-2019-0067.

[95] Jameson JL, Longo DL. Precision medicine--personalized, problematic, and promising. N Engl J Med 2015;372:2229-34. https://doi.org/10.1056/NEJMsb1503104. 
[96] Parimbelli E, Marini S, Sacchi L, Bellazzi R. Patient similarity for precision medicine: A systematic review. Journal of Biomedical Informatics 2018;83:87-96. https://doi.org/10.1016/j.jbi.2018.06.001.

[97] Kyriazakos S, Valentini V, Cesario A, Zachariae R. FORECAST - A cloud-based personalized intelligent virtual coaching platform for the well-being of cancer patients. Clinical and Translational Radiation Oncology 2018;8:50-9. https://doi.org/10.1016/j.ctro.2017.11.006.

[98] Wu HS, Gal R, van Sleeuwen NC, Brombacher AC, IJsselsteijn WA, May AM, et al. Breast Cancer Survivors' Experiences With an Activity Tracker Integrated Into a Supervised Exercise Program: Qualitative Study. JMIR Mhealth Uhealth 2019;7:e10820. https://doi.org/10.2196/10820.

[99] Roberts AL, Potts HW, Koutoukidis DA, Smith L, Fisher A. Breast, Prostate, and Colorectal Cancer Survivors' Experiences of Using Publicly Available Physical Activity Mobile Apps: Qualitative Study. JMIR Mhealth Uhealth 2019;7:e10918. https://doi.org/10.2196/10918.

[100] Trinh L, Arbour-Nicitopoulos KP, Sabiston CM, Berry SR, Loblaw A, Alibhai SMH, et al. RiseTx: testing the feasibility of a web application for reducing sedentary behavior among prostate cancer survivors receiving androgen deprivation therapy. Int J Behav Nutr Phys Act 2018;15:49. https://doi.org/10.1186/s12966-018-0686-0.

[101] Lozano-Lozano M, Cantarero-Villanueva I, Martin-Martin L, Galiano-Castillo N, Sanchez M-J, Fernández-Lao C, et al. A Mobile System to Improve Quality of Life Via Energy Balance in Breast Cancer Survivors (BENECA mHealth): Prospective Test-Retest Quasiexperimental Feasibility Study. JMIR Mhealth Uhealth 2019;7:e14136. https://doi.org/10.2196/14136.

[102] Chow PI, Drago F, Kennedy EM, Cohn WF. A Novel Mobile Phone App Intervention With Phone Coaching to Reduce Symptoms of Depression in Survivors of Women's Cancer: Pre-Post Pilot Study. JMIR Cancer 2020;6:e15750. https://doi.org/10.2196/15750.

[103] Smith SK, Kuhn E, O’Donnell J, Koontz BF, Nelson N, Molloy K, et al. Cancer distress coach: Pilot study of a mobile app for managing posttraumatic stress. Psychooncology 2018;27:350-3. https://doi.org/10.1002/pon.4363.

[104] Kubo A, Altschuler A, Kurtovich E, Hendlish S, Laurent CA, Kolevska T, et al. A Pilot Mobile-Based Mindfulness Intervention for Cancer Patients and Their Informal Caregivers. Mindfulness 2018;9:1885-94. https://doi.org/10.1007/s12671-018-0931-2.

[105] Wiklander M, Strandquist J, Obol CM, Eriksson LE, Winterling J, Rodriguez-Wallberg KA, et al. Feasibility of a self-help web-based intervention targeting young cancer patients with sexual problems and fertility distress. Support Care Cancer 2017;25:3675-82. https://doi.org/10.1007/s00520-017-3793-6.

[106] Beck SL, Eaton LH, Echeverria C, Mooney KH. SymptomCare@Home: Developing an Integrated Symptom Monitoring and Management System for Outpatients Receiving Chemotherapy. Comput Inform Nurs 2017;35:520-9. https://doi.org/10.1097/CIN.0000000000000364.

[107] Kalke K, Ginossar T, Bentley JM, Carver H, Shah SFA, Kinney AY. Use of EvidenceBased Best Practices and Behavior Change Techniques in Breast Cancer Apps: Systematic Analysis. JMIR Mhealth Uhealth 2020;8:e14082. https://doi.org/10.2196/14082.

[108] Vollmer Dahlke D, Fair K, Hong YA, Beaudoin CE, Pulczinski J, Ory MG. Apps seeking theories: results of a study on the use of health behavior change theories in cancer 
survivorship mobile apps. JMIR Mhealth Uhealth 2015;3:e31.

https://doi.org/10.2196/mhealth.3861.

[109] Abraham C, Michie S. A taxonomy of behavior change techniques used in interventions. Health Psychology 2008;27:379-87. https://doi.org/10.1037/0278-6133.27.3.379.

[110] Jibb LA, Stevens BJ, Nathan PC, Seto E, Cafazzo JA, Johnston DL, et al. Implementation and preliminary effectiveness of a real-time pain management smartphone app for adolescents with cancer: A multicenter pilot clinical study. Pediatr Blood Cancer 2017;64. https://doi.org/10.1002/pbc.26554.

[111] Falchook AD, Tracton G, Stravers L, Fleming ME, Snavely AC, Noe JF, et al. Use of mobile device technology to continuously collect patient-reported symptoms during radiation therapy for head and neck cancer: A prospective feasibility study. Adv Radiat Oncol 2016;1:115-21. https://doi.org/10.1016/j.adro.2016.02.001.

[112] Peltola MK, Lehikoinen JS, Sippola LT, Saarilahti K, Mäkitie AA. A Novel Digital Patient-Reported Outcome Platform for Head and Neck Oncology Patients-A Pilot Study. Clin Med Insights Ear Nose Throat 2016;9:1-6. https://doi.org/10.4137/CMENT.S40219.

[113] Warrington L, Absolom K, Holch P, Gibson A, Clayton B, Velikova G. Online tool for monitoring adverse events in patients with cancer during treatment (eRAPID): field testing in a clinical setting. BMJ Open 2019;9:e025185. https://doi.org/10.1136/bmjopen-2018025185 .

[114] Cvetković J. Breast Cancer Patients’ Depression Prediction by Machine Learning Approach. Cancer Invest 2017;35:569-72. https://doi.org/10.1080/07357907.2017.1363892.

[115] Ganggayah MD, Taib NA, Har YC, Lio P, Dhillon SK. Predicting factors for survival of breast cancer patients using machine learning techniques. BMC Med Inform Decis Mak 2019;19:48. https://doi.org/10.1186/s12911-019-0801-4.

[116] Tian Y, Li J, Zhou T, Tong D, Chi S, Kong X, et al. Spatially varying effects of predictors for the survival prediction of nonmetastatic colorectal Cancer. BMC Cancer 2018;18:1084. https://doi.org/10.1186/s12885-018-4985-2.

[117] Gupta P, Chiang S-F, Sahoo PK, Mohapatra SK, You J-F, Onthoni DD, et al. Prediction of Colon Cancer Stages and Survival Period with Machine Learning Approach. Cancers (Basel) 2019;11. https://doi.org/10.3390/cancers11122007.

[118] Tan MS, Tan JW, Chang S-W, Yap HJ, Abdul Kareem S, Zain RB. A genetic programming approach to oral cancer prognosis. PeerJ 2016;4:e2482. https://doi.org/10.7717/peerj.2482.

[119] Pota M, Scalco E, Sanguineti G, Farneti A, Cattaneo GM, Rizzo G, et al. Early prediction of radiotherapy-induced parotid shrinkage and toxicity based on CT radiomics and fuzzy classification. Artif Intell Med 2017;81:41-53. https://doi.org/10.1016/j.artmed.2017.03.004.

[120] Zylla DM, Gilmore GE, Steele GL, Eklund JP, Wood CM, Stover AM, et al. Collection of electronic patient-reported symptoms in patients with advanced cancer using Epic MyChart surveys. Support Care Cancer 2019. https://doi.org/10.1007/s00520-019-05109-0.

[121] Fallahzadeh R, Rokni SA, Ghasemzadeh H, Soto-Perez-de-Celis E, Shahrokni A. Digital Health for Geriatric Oncology. JCO Clin Cancer Inform 2018;2:1-12. https://doi.org/10.1200/CCI.17.00133.

[122] Duman-Lubberding S, van Uden-Kraan CF, Jansen F, Witte BI, Eerenstein SEJ, van Weert $S$, et al. Durable usage of patient-reported outcome measures in clinical practice to 
monitor health-related quality of life in head and neck cancer patients. Support Care Cancer 2017;25:3775-83. https://doi.org/10.1007/s00520-017-3808-3.

[123] Karadaghy OA, Shew M, New J, Bur AM. Development and Assessment of a Machine Learning Model to Help Predict Survival Among Patients With Oral Squamous Cell Carcinoma. JAMA Otolaryngol Head Neck Surg 2019. https://doi.org/10.1001/jamaoto.2019.0981.

[124] Collado-Borrell R, Escudero-Vilaplana V, Ribed A, Anglada-Martínez H, Martín-Conde M, Herranz-Alonso A, et al. Design of the functional requirements of a smartphone app for patients receiving oral antineoplastic agents: The e-OncoSalud® app. J Oncol Pharm Pract 2020;26:105-15. https://doi.org/10.1177/1078155219840419.

[125] Holch P, Warrington L, Bamforth LCA, Keding A, Ziegler LE, Absolom K, et al. Development of an integrated electronic platform for patient self-report and management of adverse events during cancer treatment. Ann Oncol 2017;28:2305-11. https://doi.org/10.1093/annonc/mdx317.

[126] Girgis A, Durcinoska I, Levesque JV, Gerges M, Sandell T, Arnold A, et al. eHealth System for Collecting and Utilizing Patient Reported Outcome Measures for Personalized Treatment and Care (PROMPT-Care) Among Cancer Patients: Mixed Methods Approach to Evaluate Feasibility and Acceptability. J Med Internet Res 2017;19:e330. https://doi.org/10.2196/jmir.8360.

[127] Fortier MA, Chung WW, Martinez A, Gago-Masague S, Sender L. Pain buddy: A novel use of m-health in the management of children's cancer pain. Comput Biol Med 2016;76:202-14. https://doi.org/10.1016/j.compbiomed.2016.07.012.

[128] Fishbein JN, Nisotel LE, MacDonald JJ, Amoyal Pensak N, Jacobs JM, Flanagan C, et al. Mobile Application to Promote Adherence to Oral Chemotherapy and Symptom Management: A Protocol for Design and Development. JMIR Res Protoc 2017;6:e62. https://doi.org/10.2196/resprot.6198.

[129] Moradian S, Krzyzanowska MK, Maguire R, Morita PP, Kukreti V, Avery J, et al. Usability Evaluation of a Mobile Phone-Based System for Remote Monitoring and Management of Chemotherapy-Related Side Effects in Cancer Patients: Mixed-Methods Study. JMIR Cancer 2018;4:e10932. https://doi.org/10.2196/10932.

[130] Oldenmenger WH, Witkamp FE, Bromberg JEC, Jongen JLM, Lieverse PJ, Huygen FJPM, et al. To be in pain (or not): a computer enables outpatients to inform their physician. Ann Oncol 2016;27:1776-81. https://doi.org/10.1093/annonc/mdw250.

[131] Maguire R, Fox PA, McCann L, Miaskowski C, Kotronoulas G, Miller M, et al. The eSMART study protocol: a randomised controlled trial to evaluate electronic symptom management using the advanced symptom management system (ASyMS) remote technology for patients with cancer. BMJ Open 2017;7:e015016. https://doi.org/10.1136/bmjopen-2016-015016.

[132] Schera F, Schäfer M, Bucur A, van Leeuwen J, Ngantchjon EH, Graf N, et al. iManageMyHealth and iSupportMyPatients: mobile decision support and health management apps for cancer patients and their doctors. Ecancermedicalscience 2018;12:848. https://doi.org/10.3332/ecancer.2018.848.

[133] Mooney K, Whisenant MS, Beck SL. Symptom Care at Home: A Comprehensive and Pragmatic PRO System Approach to Improve Cancer Symptom Care. Med Care 2019;57 Suppl 5 Suppl 1:S66-72. https://doi.org/10.1097/MLR.0000000000001037. 
[134] Piau A, Crissey R, Brechemier D, Balardy L, Nourhashemi F. A smartphone Chatbot application to optimize monitoring of older patients with cancer. Int $\mathrm{J}$ Med Inform 2019;128:18-23. https://doi.org/10.1016/j.ijmedinf.2019.05.013.

[135] Furlong E, Darley A, Fox P, Buick A, Kotronoulas G, Miller M, et al. Adaptation and Implementation of a Mobile Phone-Based Remote Symptom Monitoring System for People With Cancer in Europe. JMIR Cancer 2019;5:e10813. https://doi.org/10.2196/10813.

[136] Sasani K, Catanese HN, Ghods A, Rokni SA, Ghasemzadeh H, Downey RJ, et al. Gait speed and survival of older surgical patient with cancer: Prediction after machine learning. J Geriatr Oncol 2019;10:120-5. https://doi.org/10.1016/j.jgo.2018.06.012.

[137] Sena GR, Lima TPF, Mello MJG, Thuler LCS, Lima JTO. Developing Machine Learning Algorithms for the Prediction of Early Death in Elderly Cancer Patients: Usability Study. JMIR Cancer 2019;5:e12163. https://doi.org/10.2196/12163.

[138] Ji W, Kwon H, Lee S, Kim S, Hong JS, Park YR, et al. Mobile Health Management Platform-Based Pulmonary Rehabilitation for Patients With Non-Small Cell Lung Cancer: Prospective Clinical Trial. JMIR Mhealth Uhealth 2019;7:e12645. https://doi.org/10.2196/12645.

[139] He J, Zhang JX, Chen C-T, Ma Y, De Guzman R, Meng J, et al. The Relative Importance of Clinical and Socio-Demographic Variables in Prognostic Prediction in Non-Small Cell Lung Cancer: A Variable Importance Approach. Med Care 2020. https://doi.org/10.1097/MLR.0000000000001288.

[140] Wong NC, Lam C, Patterson L, Shayegan B. Use of machine learning to predict early biochemical recurrence after robot-assisted prostatectomy. BJU Int 2019;123:51-7. https://doi.org/10.1111/bju.14477.

[141] Obrzut B, Kusy M, Semczuk A, Obrzut M, Kluska J. Prediction of 5-year overall survival in cervical cancer patients treated with radical hysterectomy using computational intelligence methods. BMC Cancer 2017;17:840. https://doi.org/10.1186/s12885-017-38063.

[142] Eminaga O, Al-Hamad O, Boegemann M, Breil B, Semjonow A. Combination possibility and deep learning model as clinical decision-aided approach for prostate cancer. Health Informatics J 2019:1460458219855884. https://doi.org/10.1177/1460458219855884.

[143] Cosma G, Acampora G, Brown D, Rees RC, Khan M, Pockley AG. Prediction of Pathological Stage in Patients with Prostate Cancer: A Neuro-Fuzzy Model. PLoS ONE 2016;11:e0155856. https://doi.org/10.1371/journal.pone.0155856.

[144] Peleg M. Computer-interpretable clinical guidelines: A methodological review. Journal of Biomedical Informatics 2013;46:744-63. https://doi.org/10.1016/j.jbi.2013.06.009.

[145] Xu Y, Ju L, Tong J, Zhou C, Yang J. Supervised Machine Learning Predictive Analytics For Triple-Negative Breast Cancer Death Outcomes. Onco Targets Ther 2019;12:9059-67. https://doi.org/10.2147/OTT.S223603.

[146] Peleg M, Keren S, Denekamp Y. Mapping computerized clinical guidelines to electronic medical records: Knowledge-data ontological mapper (KDOM). Journal of Biomedical Informatics 2008;41:180-201. https://doi.org/10.1016/j.jbi.2007.05.003.

[147] German E, Leibowitz A, Shahar Y. An architecture for linking medical decision-support applications to clinical databases and its evaluation. Journal of Biomedical Informatics 2009;42:203-18. https://doi.org/10.1016/j.jbi.2008.10.007. 
[148] Prochaska JJ, Coughlin SS, Lyons EJ. Social Media and Mobile Technology for Cancer Prevention and Treatment. Am Soc Clin Oncol Educ Book 2017;37:128-37. https://doi.org/10.1200/EDBK_173841.

[149] Robertson MC, Tsai E, Lyons EJ, Srinivasan S, Swartz MC, Baum ML, et al. Mobile Health Physical Activity Intervention Preferences in Cancer Survivors: A Qualitative Study. JMIR Mhealth Uhealth 2017;5:e3. https://doi.org/10.2196/mhealth.6970.

[150] Ginossar T, Shah SFA, West AJ, Bentley JM, Caburnay CA, Kreuter MW, et al. Content, Usability, and Utilization of Plain Language in Breast Cancer Mobile Phone Apps: A Systematic Analysis. JMIR Mhealth Uhealth 2017;5:e20. https://doi.org/10.2196/mhealth.7073.

[151] Han JY, Hawkins R, Baker T, Shah DV, Pingree S, Gustafson DH. How Cancer Patients Use and Benefit from an Interactive Cancer Communication System. J Health Commun 2017;22:792-9. https://doi.org/10.1080/10810730.2017.1360413.

[152] Rahm AK, Hawkins RP, Dearing JW, Pingree S, Lomax JB, McDowell H, et al. Implementing an evidence-based breast cancer support and communication tool to newly diagnosed patients as standard care in two institutions. Transl Behav Med 2015;5:198-206. https://doi.org/10.1007/s13142-015-0305-4. 


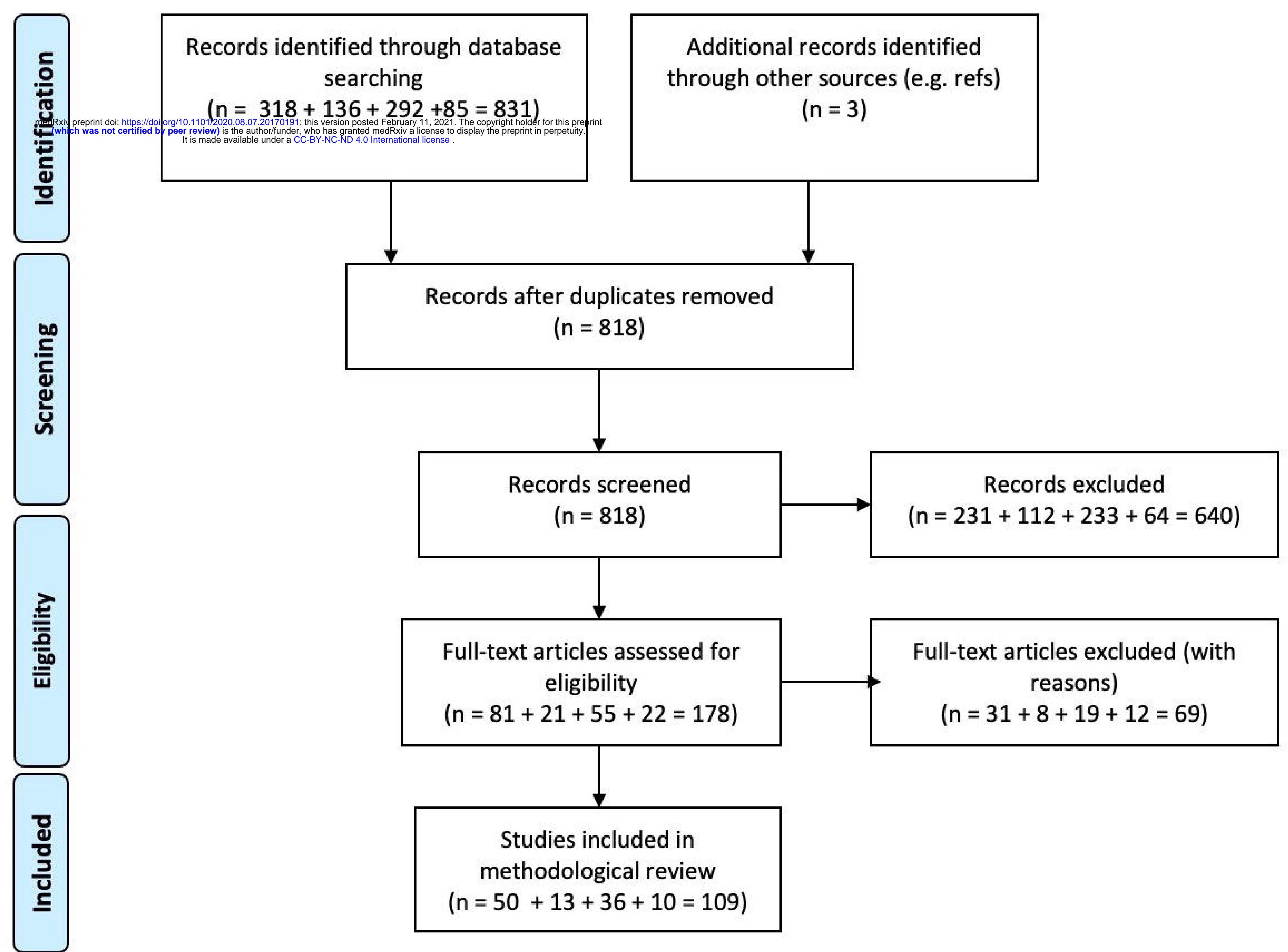




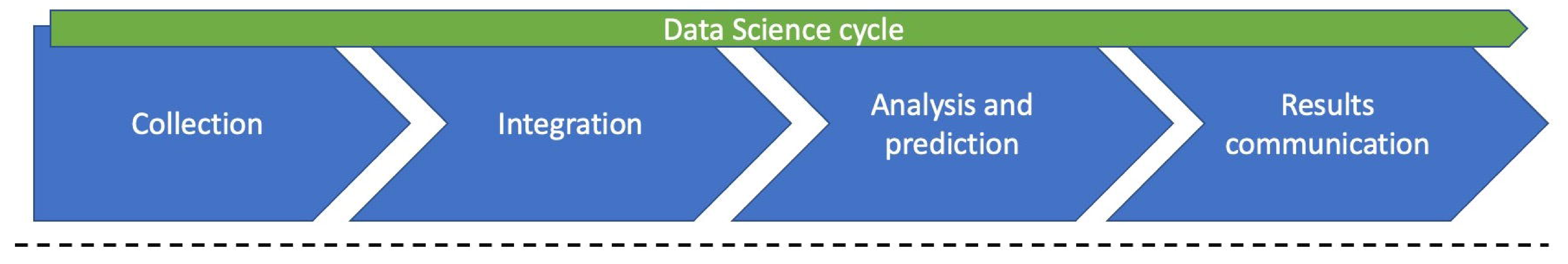

data generation and collection processing, integration, storage, management predictive modeling

visualization, interpretation and actionable outcomes 